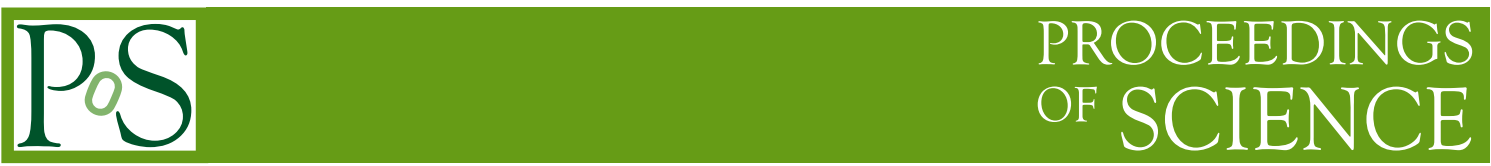

\title{
An Introduction to Nonassociative Physics
}

\section{Richard J. Szabo*}

Department of Mathematics, Heriot-Watt University, Edinburgh, United Kingdom. Maxwell Institute for Mathematical Sciences, Edinburgh, United Kingdom.

The Higgs Centre for Theoretical Physics, Edinburgh, United Kingdom.

E-mail: R.J.Szabo@hw.ac.uk

We give a pedagogical introduction to the nonassociative structures arising from recent developments in quantum mechanics with magnetic monopoles, in string theory and M-theory with nongeometric fluxes, and in M-theory with non-geometric Kaluza-Klein monopoles. After a brief overview of the main historical appearences of nonassociativity in quantum mechanics, string theory and M-theory, we provide a detailed account of the classical and quantum dynamics of electric charges in the backgrounds of various distributions of magnetic charge. We apply Born reciprocity to map this system to the phase space of closed strings propagating in $R$-flux backgrounds of string theory, and then describe the lift to the phase space of M2-branes in $R$-flux backgrounds of M-theory. Applying Born reciprocity maps this M-theory configuration to the phase space of M-waves probing a non-geometric Kaluza-Klein monopole background. These four perspective systems are unified by a covariant 3-algebra structure on the M-theory phase space.

Corfu Summer Institute 2018 "School and Workshops on Elementary Particle Physics and Gravity" (CORFU2018)

31 August-28 September 2018

Corfu, Greece

Preprint: EMPG-19-10

\footnotetext{
* Speaker.
} 


\section{Contents}

1. A brief history of nonassociativity in physics 2

$\begin{array}{lll}1.1 & \text { Nonassociative algebra } & 2\end{array}$

1.2 The octonions 2

1.3 Jordanian quantum mechanics 4

1.4 Nambu mechanics 5

$\begin{array}{ll}1.5 & \text { Nonassociativity in string theory }\end{array}$

1.6 Nonassociativity in M-theory 8

$\begin{array}{lll}1.7 \text { Outline } & 10\end{array}$

2. Electric charges in magnetic monopole backgrounds $\quad 11$

2.1 Magnetic Poisson brackets 11

2.2 Magnetic monopoles 12

$\begin{array}{lll}2.3 & \text { Classical motion in fields of magnetic charge } & 14\end{array}$

2.4 Quantization of magnetic Poisson brackets 16

$\begin{array}{ll}2.5 & \text { Nonassociative quantum mechanics } \\ & 20\end{array}$

3. Closed strings in locally non-geometric flux backgrounds 23

$\begin{array}{lll}3.1 & \text { The } R \text {-flux model } & 23\end{array}$

3.2 How closed strings see nonassociativity 25

$\begin{array}{lll}3.3 & \text { Nonassociative gravity } & 26\end{array}$

4. M2-branes in locally non-geometric flux backgrounds 27

$4.1 \quad$ M-theory lift of the $R$-flux model 27

4.2 Quantization of the M2-brane phase space 29

5. M-waves in non-geometric Kaluza-Klein monopole backgrounds 31

$\begin{array}{lll}5.1 & \text { Magnetic monopoles in quantum gravity } & 31\end{array}$

$\begin{array}{lll}5.2 & \text { The M-wave phase space } & 32\end{array}$

5.3 The covariant M-theory phase space 3-algebra 33 


\section{A brief history of nonassociativity in physics}

\subsection{Nonassociative algebra}

Nonassociative algebras first appeared around the middle of the nineteenth century and have since formed an independent branch of mathematics. By far the simplest and best known example of a nonassociative algebra is a Lie algebra. A Lie bracket $[\cdot, \cdot]$ on a vector space is generally a noncommutative and nonassociative binary operation in the sense that $[x, y] \neq[y, x]$ and $[x,[y, z]] \neq$ $[[x, y], z]$, but rather it is antisymmetric and satisfies the Jacobi identity. If we package the quantity which vanishes by the Jacobi identity into a ternary operation called the 'Jacobiator',

$$
[x, y, z]:=\frac{1}{3}([x,[y, z]]-[[x, y], z]+[[x, z], y]),
$$

then Lie algebras are characterized by the feature that this 3-bracket vanishes: $[x, y, z]=0$. In our present context this will be considered to be a trivial example of a nonassociative algebra, as the binary operation on the Lie group which integrates the Lie algebra via the exponential map is associative. In fact, in this paper we will be looking at cases of algebras with a multiplication that has non-vanishing Jacobiators, just like the multiplication in a noncommutative algebra can be characterized by a non-vanishing commutator $[x, y]:=x y-y x \neq 0$. A well-known example of a noncommutative and nonassociative algebra in this sense is the algebra of octonions, which constitutes an important example of an 'alternative algebra', where associativity of the multiplication (and hence the Jacobi identity) is generally violated, but it possesses the alternativity condition which generalizes the Jordan identity $\left(x^{2} y\right) x=x^{2}(y x)$ of (commutative) Jordan algebras, which we review below.

Algebras whose associativity is controlled by identities like the Jacobi or alternative identities were of central mathematical interest in the last century, as arbitrarily relaxing associativity in an algebra does not lead to a good mathematical theory unless some other structures are present, see e.g. [1]. They have recently made their way into the forefront of some recent developments in quantum mechanics, string theory and M-theory. The main aim of these lectures is to survey some of these recent insights and to highlight some of the interesting physical consequences of the theory, together with the many important open avenues awaiting further investigation.

Nonassociative algebras actually have a long and diverse history of appearences in physics, which is perhaps not so widely appreciated. The purpose of this opening section is to briefly review some of their occurences with a personally biased point of view: We only discuss developments in physics with an eye to the main topics that we will pursue later on, and to overview the developments that we shall treat in more detail in subsequent sections.

\subsection{The octonions}

For later use, let us begin by recalling the standard mathematical example of the octonions, as it will play an important role in some of the discussions throughout this paper (see e.g. [2] for a nice introduction). A well-known theorem in algebra states that there are only four normed division algebras over the field $\mathbb{R}$ of real numbers: The real numbers $\mathbb{R}$ themselves, the complex numbers $\mathbb{C}$, the quaternions $\mathbb{H}$, and the octonions $\mathbb{O}$. Both $\mathbb{R}$ and $\mathbb{C}$ are commutative and associative algebras, $\mathbb{H}$ is noncommutative but associative, while $\mathbb{O}$ is both noncommutative and nonassociative. The 
algebra $\mathbb{R}$ is generated over itself by a central identity element $1, \mathbb{C}$ is generated over $\mathbb{R}$ by 1 and an imaginary complex unit $i=\sqrt{-1}$, while $\mathbb{H}$ is generated by 1 and three imaginary quaternion units $\mathrm{i}, \mathrm{j}$ and $\mathrm{k}$, familar from quantum mechanics in their representation in terms of Pauli spin matrices $e_{i}=\sqrt{-1} \sigma_{i}, i=1,2,3$, which obey $e_{i}^{2}=-1$ and

$$
e_{i} e_{j}=-\delta_{i j}+\varepsilon_{i j k} e_{k}
$$

where $\varepsilon_{i j k}$ is the Levi-Civita symbol in three dimensions.

Analogously, the algebra $\mathbb{O}$ is generated by 1 and seven imaginary octonion units $e_{A}, A=$ $1, \ldots, 7$, satisfying $e_{A}^{2}=-1$, so that a generic element $O$ of $\mathbb{O}$ is of the form

$$
O=a_{0}+a_{1} e_{1}+a_{2} e_{2}+\cdots+a_{7} e_{7}
$$

with $a_{0}, a_{1}, \ldots, a_{7} \in \mathbb{R}$. The multiplication in the algebra $\mathbb{O}$ can be written in the form

$$
e_{A} e_{B}=-\delta_{A B}+\eta_{A B C} e_{C}
$$

where the structure constants $\eta_{A B C}$ form a completely antisymmetric tensor with only seven nonvanishing components, generalizing the structure constants $\varepsilon_{i j k}$ (with only one non-zero component) of the algebra of quaternions $\mathbb{H}$.

To specify $\eta_{A B C}$ explicitly, it is convenient to rewrite $e_{4}, e_{5}, e_{6}=f_{1}, f_{2}, f_{3}$ and represent the algebra of octonions in terms of the commutators

$$
\begin{array}{ll}
{\left[e_{i}, e_{j}\right]=2 \varepsilon_{i j k} e_{k} \quad \text { and } \quad\left[e_{7}, e_{i}\right]=2 f_{i},} \\
{\left[f_{i}, f_{j}\right]=-2 \varepsilon_{i j k} e_{k} \quad \text { and } \quad\left[e_{7}, f_{i}\right]=-2 e_{i},} \\
{\left[e_{i}, f_{j}\right]=2\left(\delta_{i j} e_{7}-\varepsilon_{i j k} f_{k}\right) .}
\end{array}
$$

The first set of commutation relations describes a quaternion subalgebra $\mathbb{H} \subset \mathbb{O}$ (the Lie algebra of $S U(2) \simeq S O(3)$ ), analogous to the hierarchy of embeddings $\mathbb{R} \subset \mathbb{C} \subset \mathbb{H}$ generated by the inclusions of 1 and $i$; this follows from the Cayley-Dickson construction of the real normed division algebras. This demonstrates the important feature that, unlike $\mathbb{H}$ whose structure constants are invariant under the full three-dimensional rotation group $S O(3)$, the symmetry group of the tensor $\eta_{A B C}$ is only the 14-dimensional simple exceptional Lie subgroup $G_{2} \subset S O(7)$ of the rotation group in seven dimensions. Using these commutators one can derive the non-vanishing Jacobiators

$$
\left[e_{A}, e_{B}, e_{C}\right]=-4 \eta_{A B C D} e_{D}
$$

with antisymmetric structure constants $\eta_{A B C D}$ also having only seven non-vanishing components. The alternative property of the algebra $\mathbb{O}$ is then equivalent to the statement that its Jacobiators are proportional to its 'associators':

$$
\left[e_{A}, e_{B}, e_{C}\right]=2\left(\left(e_{A} e_{B}\right) e_{C}-e_{A}\left(e_{B} e_{C}\right)\right)
$$




\subsection{Jordanian quantum mechanics}

The algebra of octonions $\mathbb{O}$ is an example of what may be called a noncommutative Jordan algebra, which we now proceed to define. Jordan algebras were the first appearence of nonassociativity in physics, in the early days of quantum theory [3]. Jordan's motivation for introducing them was to define an algebraic structure on the set of observables in quantum mechanics, and hence an overall new algebraic setting for the foundations of quantum theory. The observables of any quantum system are given by Hermitian elements in a $C^{*}$-algebra, which can be represented by operators on a finite- or infinite-dimensional separable Hilbert space. If $A$ and $B$ are Hermitian operators, then their operator product is not a Hermitian operator (unless they commute), and neither is their commutator. Thus neither of these binary operations closes on the set of physical observables, and so they are not an intrinsic part of the physically meaningful characteristics of the system. Jordan algebras were introduced to formalize the properties of a finite-dimensional quantum system.

The basic observation is that the symmetrized product

$$
A \circ B=\frac{1}{2}(A B+B A)=\frac{1}{2}\left((A+B)^{2}-A^{2}-B^{2}\right)
$$

is Hermitian. This product is obviously commutative,

$$
A \circ B=B \circ A
$$

but it is nonassociative: $(A \circ B) \circ C \neq A \circ(B \circ C)$ in general. However, it satisfies the Jordan identity

$$
\left(A^{2} \circ B\right) \circ A=A^{2} \circ(B \circ A)
$$

which is equivalent to the power-associativity property

$$
A^{n} \circ A^{m}=A^{n+m}
$$

for all non-negative integer powers $n$ and $m$. Hermitian operators also have the property that $A^{2}+$ $B^{2}=0$ implies $A=B=0$, i.e. the symmetrized product on observables is formally real.

Abstracting these properties leads to the notion of a Jordan algebra as a commutative powerassociative algebra; in other words, although the set of observables do not form an algebra under the usual multiplication of operators, they do form a Jordan algebra with respect to the symmetrized product. If the multiplication on a Jordan algebra can be brought via a suitable isomorphism to the form of a symmetrized product $\circ$ on an associative algebra, as above, it is said to be special. The power-associative property implies that it is sufficient to demand that the binary operation on a Jordan algebra is alternative [4,5],

$$
(x y) x=x(y x) .
$$

By abstracting this property we may thus define the notion of a noncommutative Jordan algebra wherein we relax the commutativity of the multiplication.

The starting programme of Jordanian quantum mechanics was then to find examples of nonspecial Jordan algebras as algebras of observables for quantum systems. These hopes were dashed 
by the famous work of Jordan, von Neumann and Wigner [6] who proved that the only simple formally real finite-dimensional Jordan algebras are the Jordan algebras of $n \times n$ Hermitian matrices over $\mathbb{R}, \mathbb{C}$ and $\mathbb{H}$, the exceptional 27-dimensional Albert algebra of $3 \times 3$ Hermitian matrices over the octonions $\mathbb{O}$, and the Clifford-type (or 'spin') Jordan algebras; among these, only the Albert algebra, equiped with the symmetrized product, is non-special. An 'octonionic quantum mechanics' based on the Albert algebra was later developed by [7] which satisfies the von Neumann axioms of quantum theory, despite there being no Hilbert space formulation for nonassociative algebras: Operators acting on a separable Hilbert space necessarily associate. The Jordan-von NeumannWigner theorem was generalized quite some time later by Zelmanov [8] to the infinite-dimensional case. This implies that there is no infinite-dimensional non-special Jordan algebra that would accomodate the observables of quantum mechanics, thus eliminating the prospects of Jordan algebras from playing a viable role in the foundations of quantum theory.

\subsection{Nambu mechanics}

Let us now fast-forward ahead many years to a very different kind of nonassociative structure that takes place directly at the level of a ternary operation. Such structures were introduced by Nambu [9]; they were formalised and generalised 20 years later by Takhtajan [10]. A NambuPoisson bracket on a manifold $M$ is a completely antisymmetric ternary operation $\{\cdot, \cdot, \cdot\}$ on the algebra of functions on $M$ which obeys the Leibniz rule,

$$
\{f g, h, k\}=f\{g, h, k\}+\{f, h, k\} g,
$$

and a higher version of the Jacobi identity for Lie algebras called the "fundamental identity",

$$
\left\{f, g,\left\{h_{1}, h_{2}, h_{3}\right\}\right\}-\left\{\left\{f, g, h_{1}\right\}, h_{2}, h_{3}\right\}+\left\{\left\{f, g, h_{2}\right\}, h_{1}, h_{3}\right\}-\left\{\left\{f, g, h_{3}\right\}, h_{1}, h_{2}\right\}=0,
$$

for functions $f, g, h, h_{1}, h_{2}, h_{3}, k \in C^{\infty}(M)$. These axioms extend the axioms of a Poisson bracket which defines a Lie algebra on the vector space $C^{\infty}(M)$; likewise, a Nambu-Poisson bracket defines a higher version of a Lie algebra on the vector space $C^{\infty}(M)$ called a '3-Lie algebra' [10].

Nambu's idea was to reformulate the equations of motion of complicated non-integrable dynamical systems as a bi-Hamiltonian dynamics with flow equations

$$
\frac{\mathrm{d} f}{\mathrm{~d} t}=\left\{f, H_{1}, H_{2}\right\}
$$

involving a pair of Hamiltonians $H_{1}$ and $H_{2}$. The hope was to discover new (generalized) integrals of motion. Consistency of these time evolution equations necessitates both the Leibniz rule and also the fundamental identity if one requires time derivatives to act as derivations of the 3-bracket, so that they play crucial roles analogous to the roles of the Leibniz rule and Jacobi identity of the Poisson bracket in ordinary Hamiltonian mechanics.

Nambu's original example was the canonical 3-bracket on $M=\mathbb{R}^{3}$ given by

$$
\{f, g, h\}:=\varepsilon^{i j k} \partial_{i} f \partial_{j} g \partial_{k} h,
$$

and the motivating dynamical problem is given by the Euler equations

$$
\frac{\mathrm{d} \vec{L}}{\mathrm{~d} t}=\vec{L} \times \vec{\omega}
$$


describing the motion of a rotating rigid body in $\mathbb{R}^{3}$ with orbital angular momentum $\vec{L}$ and angular velocity $\vec{\omega}$ about its principal axes. Nambu's observation was that these equations are equivalent to the bi-Hamiltonian equations

$$
\frac{\mathrm{d} L_{i}}{\mathrm{~d} t}=\left\{L_{i}, \vec{L}^{2}, T\right\}
$$

for the components $L_{i}$ of the angular momentum with respect to the 3-bracket (1.16) with $\partial_{i}=$ $\partial / \partial L_{i}$, and the pair of Hamiltonians $H_{1}=\vec{L}^{2}$ and $H_{2}=T=\frac{1}{2} \vec{L} \cdot \vec{\omega}$.

Attempts to quantize the Nambu-Poisson bracket brings us closer to our earlier discussions of nonassociativity. If one wishes to use a correspondence principle analogous to the usual Bohr correspondence principle of quantum mechanics, then the natural extension of quantizing Poisson brackets, by mapping them to commutators of operators on a Hilbert space, would be to map Nambu-Poisson brackets to Jacobiators, but this does not work because operators on a separable Hilbert space always associate and so their Jacobiators vanish, as mentioned before. Instead, one can define the Nambu-Heisenberg bracket to be the "half-Jacobiator" [9]

$$
[A, B, C]_{\mathrm{NH}}=[A, B] C-[A, C] B+[B, C] A
$$

consisting of an antisymmetric combination of half of all terms which would otherwise vanish by the Jacobi identity. This proposal unfortunately meets with several problems, most notably that the fundamental identity is not preserved under the correspondence principle. There is a tension between the Leibniz rule and the fundamental identity as, in contrast to the Jacobi identity satisfied by a Poisson bracket, the fundamental identity imposes algebraic in addition to differential constraints on the allowed Nambu-Poisson brackets. Hence it is difficult to find a suitable 3-Lie algebra of operators that would quantize the Nambu-Poisson 3-bracket of functions. In this sense the quantization of Nambu-Poisson brackets to this day remains an open problem; see [11] for a more modern perspective on this problem within our present context, together with further references.

In light of these issues, Nambu suggested to use nonassociative algebras to quantize his 3bracket, which we can then do via the Jacobiator defined in (1.1) instead of the less natural halfJacobiator. These arguments thus demonstrate that the problem of quantizing Nambu mechanics is intimately related to formulating nonassociative quantum mechanics. This perspective will be the driving theme throughout the rest of this article.

\subsection{Nonassociativity in string theory}

String theory has been known for a long time to lead to new notions of geometry which have driven many developments and fields of mathematics. As a candidate quantum theory of gravity, it also offers a host of modifications and deformations of the notion of spacetime itself. Nonassociative structures in string theory have a relatively long history of appearences predating the more modern developments that we shall discuss in subsequent sections, which is again perhaps not so widely appreciated. Here we shall highlight some of the most important selected examples of nonassociativity in the context of string theory.

Closed string field theory. Noncommutative geometry first made its appearence in string theory in the mid-1980s with Witten's work on bosonic open string field theory [12]. Shortly thereafter, 
nonassociativity originally appeared in string theory through Strominger's work [13] which showed that the algebraic structures of gauge symmetries underlying bosonic closed string field theory do not possess the usual algebraic features of conventional symmetries in quantum field theory and string theory, which are governed infinitesimally by Lie algebras. The gauge symmetries of the theory include local spacetime diffeomorphisms, and the gauge-invariant action is constructed as the associator of Witten's open string field star product. Whereas open string states associate, the associator is non-vanishing on closed string states due to anomalies in associativity [14], which nonetheless still leads to a consistent theory. The complete analysis of the gauge symmetries was carried out later on by Zwiebach [15] using the Batalin-Vilkovisky formalism, who showed that the full action of closed string field theory is defined by an infinite chain of higher string field products on the off-shell state space satisfying homotopy deformations of Jacobi-type identities. Such a structure was identified as a strong homotopy Lie algebra [16,17], or $L_{\infty}$-algebra for short, and has been realised in the mathematics community to be the most natural and general higher version of a Lie algebra which generically exhibits an infinite hierarchy of nonassociative $n$-ary operations. See [18] for a recent survey of the developments, together with both old and new applications in string theory.

D-branes in curved backgrounds. Witten's inception of noncommutative geometry in open string field theory was reinvigorated in a much simpler and precise setting over ten years later through the seminal work of Seiberg and Witten [19]. They showed that, in a particular scaling limit which decouples the open and closed string modes, the worldvolume of a D-brane in the background of a constant Kalb-Ramond field $B$ experiences a noncommutative deformation of its geometry, and the low-energy effective field theory on the D-brane is a noncommutative version of Yang-Mills theory, a non-local gauge theory which retains stringy features in the framework of quantum field theory, such as an exact open string T-duality invariance. This sparked the flurry of exciting activity in the physics community that has evolved into the area of research dubbed 'noncommutative field theory' (these days a subject class of many high energy physics journals); see e.g. $[20,21]$ for reviews of the accelerating developments through the turn of the millenium. This development was subsequently extended to the case of non-constant 2 -form $B$-fields in [22, 23], which showed that the noncommutative star products deforming the multiplication of fields on Dbrane worldvolumes become nonassociative, where the NS-NS 3-form flux $H=\mathrm{d} B$ controls the Jacobiators of star products. As in the case of string field theory, nonassociativity in this case is an off-shell phenomenon: In the integrated on-shell open string scattering amplitudes the effects of nonassociativity (but not noncommutativity) vanish due to appropriate cyclicity properties of the nonassociative star products [24], consistently with the requirements of crossing symmetry of the worldsheet conformal field theory of open strings (which still involves ordinary quantum fields that necessarily associate); see e.g. [25] for a review of these and other developments along these lines. This physical caveat will reappear in various contexts of our discussions of nonassociativity in subsequent sections. The gauge symmetries underlying these and other nonassociative Yang-Mills theories arising as decoupling limits of open string theory also do not close in the conventional way to Lie algebras, but have been recently shown to naturally form $L_{\infty}$-algebras in [26].

Topological T-duality. Around the middle of the last decade a mathematically rigorous approach 
to T-duality was developed for principal torus bundles, and also more general backgrounds with abelian isometries [27-29]. The algebra of functions in an NS-NS $H$-flux background can be described by a mildly noncommutative 'continuous trace algebra' which incorporates the effects of $H$-flux as the Dixmier-Douady characteristic class of a gerbe, just like a Kalb-Ramond field $B$ with $\mathrm{d} B=0$ can be regarded as a background magnetic field, and 'topological T-duality' can be defined in a purely algebraic way on these functions. It was realised that when the legs of the $H$-flux are aligned in a particular way along the fibre directions on which T-duality transformations are performed, the algebra of functions can map to noncommutative and even nonassociative algebras in the T-dual background. However, the phrase 'topological T-duality' refers to the fact that such algebraic T-duality transformations are not the same as the geometric maps found in the string theory literature. In particular, one cannot recover the conventional commutative algebra of functions on the fibration when $H$-flux is turned off; rather one only recovers functions up to 'Morita equivalence', which is a "symmetry" (in the physical sense) only insofar as it provides a natural isomorphism between the corresponding K-theory groups. This was the original motivation in viewing topological T-duality as a weakened version of open string T-duality, as these K-theory groups classify the charges of D-branes in the given backgrounds. It was, however, suggested that the phenomenon should be more general and also apply to the closed string sector, i.e. not only to D-brane worldvolumes, but also to the background spacetime itself, and such algebras were argued to be global versions of the 'non-geometric' string backgrounds which have no description in conventional Riemannian geometry. These deformations of closed string theory have reemerged in more recent years in a somewhat different way which avoids the unphysical features of Morita equivalence. They will constitute some of our primary examples in subsequent sections and we shall review them in more detail there.

\subsection{Nonassociativity in M-theory}

M-theory is the as yet unknown 11-dimensional quantum theory which unifies all of the consistent 10-dimensional superstring theories, and it has likewise suggested many classical and quantum deformations of geometry. Nonassociative structures have also appeared independently and in a sense more naturally in M-theory in somewhat different fashions, also predating the more modern developments we discuss in the following, whose most important highlights we summarise here.

Multiple M2-branes. In the last decade substantial progress was made in understanding the quantum geometry probed by the membranes of M-theory, which lift the fundamental string degrees of freedom from type IIA string theory. This began with the work of Basu and Harvey [30] who proposed a lift of the D1-D3-brane system in string theory to the M2-M5-brane system in M-theory. A BPS magnetic monopole solution of maximally supersymmetric Yang-Mills theory in $3+1$ dimensions can be embedded into string theory as a collection of open D1-branes ending on a D3-brane which polarize into fuzzy 2-spheres, obtained as the well-known quantization of the canonical Poisson bracket on $S^{2}$. In the lift to M-theory, this describes a self-dual string wherein open M2-branes ending on an M5-brane polarize into fuzzy 3-spheres obtained as a putative quantization of the canonical Nambu-Poisson 3-bracket on $S^{3}$; this latter quantum geometry is still not fully understood, see [11] for a related discussion and some partial attempts. As an attempt to lift the nonabelian gauge theory which governs the low-energy description of multi- 
ple D2-branes in string theory, the famous Bagger-Lambert theory then generalized this 3-bracket structure which was proposed to underlie a nonassocative algebra in which the fields of a theory of multiple M2-branes take values [31] and it was subsequently gauged to govern higher gauge symmetries in these theories [32], initially in the form of a 3-Lie algebra (see e.g. [33] for a review with more background and further references). This appearence of 3-algebras constitutes the first explicit appearence of nonassociativity in M-theory in the sense discussed in Section 1.4; the relation between the 3-algebras occuring in the Bagger-Lambert model and the quantization of Nambu-Poisson brackets was explored in [34] through the framework of reduced models, for which explicit exact localization computations were developed in [35]. The relation between these 3-algebras and $L_{\infty}$-algebras is reviewed in e.g. [36].

M5-branes in $\boldsymbol{C}$-field backgrounds. The natural lift to M-theory of the situation in which open strings ending on a D2-brane, in the background of a constant NS-NS 2-form $B$-field of 10dimensional supergravity, is the scenario in which open M2-branes end on an M5-brane, in the background of a constant 3 -form $C$-field of 11-dimensional supergravity. Whereas the boundaries of open strings are point particles and hence probe a noncommutative worldvolume theory on a D2-brane, the boundaries of M2-branes are closed strings and hence are expected to probe a noncommutative geometry on the loop space of the M5-brane worldvolume. These expectations were confirmed explicitly in $[37,38]$, which worked out a somewhat complicated noncommutative deformation of loop space. It was proposed almost 10 years later, using the Basu-Harvey equation, that the noncommutative geometry probed by open membranes on the loop space can be more compactly and simply understood in terms of a direct nonassociative 3-algebra deformation of the M5-brane worldvolume geometry itself, akin to what appears in the Bagger-Lambert theory, and that this 3-algebra should arise from a quantization of the Nambu-Poisson 3-bracket associated to the constant $C$-field [39], in the same way that the noncommutative geometry on a D2-brane arises as a quantization of the Poisson bracket associated to a constant $B$-field. The connection between these noncommutative loop space and nonassociative worldvolume 3-algebra structures was established precisely in $[40,41]$ using transgression techniques; we will comment further about this associative loop space perspective on nonassociativity later on. Nambu-Poisson brackets, and their quantization, have appeared in various other contexts in M-theory since its inception in the mid-1990s, see e.g. [42] for a review.

$G_{2}$ and $\operatorname{Spin}(7)$ backgrounds. A somewhat more indirect and subtle appearence of nonassociativity in M-theory has emerged over the years through the well-known compactifications of 11-dimensional supergravity on Riemannian manifolds with holonomy valued in the groups $G_{2}$ or $\operatorname{Spin}(7)$, which are real Ricci-flat manifolds, see e.g. [43,44]. Recall from Section 1.2 that the exceptional Lie group $G_{2} \subset S O(7)$ is precisely the automorphism group of the algebra of octonions $\mathbb{O}$. A $G_{2}$-structure on a seven-dimensional manifold is defined locally as a 3-form using the structure constants $\eta_{A B C}$ of the commutator (1.4) of octonions which specify a nonassociative binary operation. Likewise, the structure constants $\eta_{A B C D}$ of the Jacobiator (1.6) combine with $\eta_{A B C}$ to locally define a self-dual 4-form which gives a reduction of the structure group of the oriented frame bundle of an eight-dimensional manifold to $\operatorname{Spin}(7) \subset S O(8)$. For a detailed introduction, see e.g. [45]. These $G$-structures will reappear in some of our later discussions of non-geometry 
in M-theory, though in a somewhat different context which relates them directly to non-geometric string theory.

\subsection{Outline}

Let us now come to an overview of the main topics that we shall discuss in more detail in the following. In these lectures, we will focus on two intimately related occurences of nonassociativity in physics which have recently received intensive investigation:

Magnetic monopoles. A pedagogical but rich example of nonassociative quantum mechanics, unrelated to the Jordanian framework, arises in the quantization of the dynamics of electric charges moving in the background of fields of magnetic charge. This observation is in itself not recent and dates back to the mid-1980s [46,47]. This work was inspired at the time in part to understand, in a simpler framework, the commutator anomalies that arise in certain gauge theories coupled to chiral fermions which lead to violations of the Jacobi identity among the currents generating local gauge symmetries, see e.g. [48-50]. These models also provide much simpler examples, at the level of quantum mechanics and quantum field theory, of the associativity anomalies that we discussed in Section 1.5 which were encountered in closed string field theory at around the same time. As in the setting of string field theory, it was argued that these instances still lead to sensible quantum theories when suitably interpreted. Recent developments have gone beyond these interpretations to illustrate that a nonassociative version of quantum mechanics is not only physically sensible, but also has the potential to be experimentally detected. This is the content of Section 2.

Locally non-geometric fluxes. In the course of this decade it has been suggested that closed strings should probe a noncommutative and nonassociative deformation of spacetime geometry in order to capture a global perspective on locally non-geometric flux compactifications of string theory, as originally suggested by [51], at least for those which are obtained via T-duality from geometric backgrounds with an NS-NS $H$-flux; this extends, to the closed string sector, the wellestablished case of open strings probing a noncommutative (but (on-shell) associative) deformation of the worldvolume geometry on a D-brane in the presence of a Kalb-Ramond field. This suggestion has by now been interpreted from many distinct points of view, see e.g. [52-59]. This is the content of Section 3.

The locally non-geometric backgrounds of string theory have lifts to M-theory, where it is purported that closed M2-branes probing a noncommutative and nonassociative deformation of spacetime geometry also capture locally non-geometric backgrounds, at least for four-dimensional compactifications of M-theory [60,61]. These nonassociative structures are intimately related to our discussion of the octonions from Section 1.2, and also to our discussion of the role of $G_{2}$ - and $\operatorname{Spin}(7)$-holonomy manifolds in compactifications of M-theory from Section 1.6; they are the topic of Section 4.

The noncommutative geometry of D3-branes in a constant $B$-field background is completely analogous to that of the motion of electric charges in constant magnetic fields; see e.g. [62] for an introduction. Likewise, the nonassociative geometry of non-geometric string backgrounds can also be understood from a certain "dual" perspective, in a sense explained below, which relates them to the electron-monopole system discussed above. An electric charge propagating in a distribution of 
magnetic monopoles can be embedded into string theory as a D0-brane probing a distribution of D6-branes, giving another perspective on the nonassociative deformation of the spacetime transverse to the worldvolume of the D6-branes. The lift of this D0-D6-brane system to M-theory is an M-wave probing a non-geometric Kaluza-Klein monopole background of M-theory [63], which ties all of our three main examples from Sections 2-4 together, with profound physical consequences, and is the topic of Section 5.

These lecture notes are pedagogically written and unapologetically geared at a general physics audience, without assuming any detailed requisite technical knowledge of non-geometric backgrounds or of the interesting but sophisticated mathematics of the higher structures involved. A recent review of non-geometric backgrounds most relevant to our current exposition can be found in [64]. More expository technical details on the quantization of non-geometric backgrounds, their relation to double field theory and the higher structures involved, as well as of nonassociative quantum mechanics in these contexts are found in the lecture notes [65]. The more mathematically inclined reader interested in higher structures may consult the mathematical introduction [66] to the ideas presented in the following. We will give further pointers to relevant literature as we move along.

\section{Electric charges in magnetic monopole backgrounds}

\subsection{Magnetic Poisson brackets}

The purpose of this section is to describe a very simple example of nonassociativity which arises in the classical and quantum dynamics of an electric charge in fields of magnetic charge. But we begin by descibing the underlying geometry of the kinematical problem in a general way which is also tailored to our later discussions of non-geometric string theory.

We work on the $d$-dimensional configuration space $M=\mathbb{R}^{d}$ whose phase space $\mathscr{M}=T^{*} M$ is equiped with local Darboux coordinates $X^{I}=\left(x^{i}, p_{i}\right)$, where $x^{i}$ are coordinates on $M$ and $p_{i}$ are (cotangent) momentum coordinates. The canonical Poisson brackets on $\mathscr{M}$ are defined in the usual way by the inverse of the canonical symplectic 2 -form

$$
\omega_{0}=\mathrm{d} p_{i} \wedge \mathrm{d} x^{i}
$$

Any given 2-form $B \in \Omega^{2}(M)$, not necessarily closed, will be refered to generally as a 'magnetic field' in what follows, for reasons that will become clear soon. The magnetic field twists the symplectic form $\omega_{0}$ to an almost symplectic form

$$
\omega_{B}=\omega_{0}-B
$$

where the adjective 'almost' refers to the fact that $\omega_{B}$ is nondegenerate (because $\omega_{0}$ is) but not necessarily closed: $\mathrm{d} \omega_{B}=0$ if and only if $\mathrm{d} B=0$. Its inverse $\theta_{B}=\omega_{B}^{-1}$ defines the magnetic Poisson brackets

$$
\{f, g\}_{B}=\theta_{B}^{I J} \partial_{I} f \partial_{J} g
$$


on functions $f, g \in C^{\infty}(\mathscr{M})$. On the local coordinates of phase space these brackets read as

$$
\left\{x^{i}, x^{j}\right\}_{B}=0, \quad\left\{x^{i}, p_{j}\right\}_{B}=\delta^{i}{ }_{j} \quad \text { and } \quad\left\{p_{i}, p_{j}\right\}_{B}=-B_{i j}(x),
$$

and they provide a deformation of the canonical Poisson brackets on phase space. The dynamical model based on these magnetic Poisson brackets is a generalization of the Günaydin-Zumino model [47].

The noteworthy feature here is that these brackets only fulfill the Jacobi identity $\{f, g, h\}_{B}=0$ for all $f, g, h \in C^{\infty}(\mathscr{M})$ when $\omega_{B}$ is a symplectic 2-form, i.e. when the magnetic field is closed, $\mathrm{d} B=0$. In general, we may call the 3 -form $H=\mathrm{d} B$ a 'magnetic charge', also for reasons that will become apparent very soon, and it controls the associativity of the magnetic Poisson brackets (2.3) on the phase space $\mathscr{M}$; in particular, on the local coordinates one finds that only triples of momenta can nonassociate with the nonvanishing Jacobiators

$$
\left\{p_{i}, p_{j}, p_{k}\right\}_{B}=-H_{i j k}(x) .
$$

In Poisson geometry one would thereby refer to the brackets (2.3) as H-twisted Poisson brackets $[67,68]$.

\subsection{Magnetic monopoles}

One of the main motivations for the general definition of magnetic Poisson brackets from Section 2.1 is that they govern the Hamiltonian dynamics of electrically charged particles in backgrounds of magnetic charge in classical and quantum mechanics. This is the case of $d=3$ dimensions wherein the 2-form $B$ can be written using Poincare duality in terms of a vector field $\vec{B}$ in components as $B_{i j}=e \varepsilon_{i j k} B^{k}$. In this case the magnetic Poisson brackets govern the motion of an electric charge $e$ in a static magnetic field $\vec{B}$ on $\mathbb{R}^{3}$. There are three distinct cases to consider, all of which will be described in some detail in the following.

Maxwell theory. The associative case where $\mathrm{d} B=0$ corresponds in three dimensions to the classical Maxwell theory of electromagnetism, in which there are no magnetic monopoles. In terms of the magnetic field $\vec{B}$ this case reads as

$$
\vec{\nabla} \cdot \vec{B}=0
$$

and on $M=\mathbb{R}^{3}$ it implies the existence of a globally defined magnetic vector potential $\vec{A}$ with

$$
\vec{B}=\vec{\nabla} \times \vec{A}
$$

or in general dimensions $d$ that $B=\mathrm{d} A$ for a globally defined 1-form $A$.

Dirac monopoles. Dirac's semiclassical modification of Maxwell's theory postulates a magnetic field $\vec{B}_{g}$ on $\mathbb{R}^{3}$ which is sourced by a point magnetic charge $g$ at the origin of $\mathbb{R}^{3}$. This modifies the Maxwell equation (2.6) to

$$
\vec{\nabla} \cdot \vec{B}_{g}=4 \pi g \delta^{(3)}(\vec{x})
$$


This describes a singular distribution of magnetic charge which, by removing the location of the magnetic monopole, can be solved by the magnetic field on $M^{\times}=\mathbb{R}^{3} \backslash\{\overrightarrow{0}\}$ given by

$$
\vec{B}_{g}=g \frac{\vec{x}}{|\vec{x}|^{3}} .
$$

On $M^{\times}$one can write the Dirac monopole field in terms of a locally defined magnetic vector potential $\vec{A}_{g, \vec{n}}$,

$$
\vec{B}_{g}=\vec{\nabla} \times \vec{A}_{g, \vec{n}} \quad \text { with } \quad \vec{A}_{g, \vec{n}}=\frac{g}{|\vec{x}|} \frac{\vec{x} \times \vec{n}}{|\vec{x}|-\vec{x} \cdot \vec{n}}
$$

which has additional singularities along the infinite line in the direction of a fixed unit vector $\vec{n}$, which is the celebrated Dirac string singularity. Note that the Jacobiator of the magnetic Poisson brackets in this case vanishes on $M^{\times}$.

Magnetic monopoles are hypothetical particles with a single magnetic pole whose existence would explain the quantization of electric charge, as we discuss below. In spite of their vast interest in theoretical physics, they have not yet been observed with full success in experiment. They were first observed in analog condensed matter systems over ten years ago where they appear as emergent states of matter $[69,70]$. In these experiments, certain rare earth elements with the structure of a spin ice pyrocholore lattice, consisting of an array of tetrahedra with magnetic dipoles arranged at the corner atoms such that the total magnetic charge through each tetrahedron vanishes (Figure 1), are subjected to an external magnetic field and scatter neutrons, which have their own magnetic dipole moment. Local configurations of monopoles form and the resulting Dirac strings can be observed through the neutron scattering interference patterns; see [71] for further details in the context of this paper together with more references to the most important experiments.

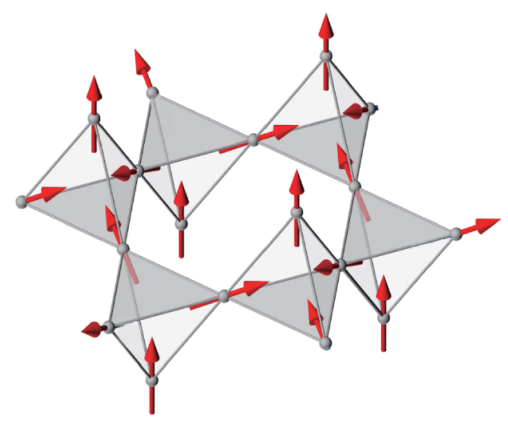

Figure 1: Spin ice pyrochlore lattice with magnetic dipoles.

Smooth sources of magnetic charge. In this article we are primarily interested in the case of nonsingular monopole distributions, wherein $\vec{\nabla} \cdot \vec{B} \neq 0$ is a smooth function, or in general dimensions $d$ the 3-form $H=\mathrm{d} B \neq 0$ is smooth; in this instance the Jacobiator of the magnetic Poisson brackets in non-vanishing throughout the smooth submanifold supporting the magnetic charge distribution. These are the cases of interest in our later discussions of nonassociativity in string theory and Mtheory, but we shall also describe other potential physical and even observable consequences of 
such models. We shall be particularly interested in the cases of uniform distributions of magnetic charge, for which $\vec{\nabla} \cdot \vec{B}=\rho$ is constant, as these are the cases which are typically amenable to exact analytic solution; then nonassociativity persists throughout all space.

\subsection{Classical motion in fields of magnetic charge}

Although we are primarily interested in the quantum mechanics of electric charge in monopole backgrounds, we can gain a lot of physical intuition about the qualitative differences between the different types of sources of magnetic charge, discussed in Section 2.2, by first discussing the classical mechanics. In $d=3$ dimensions, the motion of an electric charge $e$ with mass $m$ in the background of a static magnetic field $\vec{B}$ (with or without sources) is governed by the Lorentz force law

$$
\frac{\mathrm{d} \vec{p}}{\mathrm{~d} t}=\frac{e}{m} \vec{p} \times \vec{B}
$$

for the kinematical momentum

$$
\vec{p}=m \frac{\mathrm{d} \vec{x}}{\mathrm{~d} t}
$$

of the electron. We ignore the effects of magnetic backreaction due to the acceleration of the electric charge. Using the magnetic Poisson brackets (2.4), these equations of motion are equivalent to Hamilton's equations

$$
\frac{\mathrm{d} X^{I}}{\mathrm{~d} t}=\left\{X^{I}, \mathrm{H}\right\}_{B}
$$

for the Hamiltonian

$$
\mathrm{H}=\frac{1}{2 m} \vec{p}^{2}
$$

The particular behaviour of the trajectories determined by these dynamical evolution equations depends drastically on the nature of the magnetic field $\vec{B}$.

Uniform magnetic fields. The textbook example of a source-free background with $\vec{\nabla} \cdot \vec{B}=0$ is given by a constant magnetic field $\vec{B}$ on $\mathbb{R}^{3}$. In this case the classical dynamics is integrable and the motion is uniform along the direction of the magnetic field. The equations of motion reduce to those of a two-dimensional harmonic oscillator, with angular frequency the Larmor frequency $e|\vec{B}| / m$, in the plane perpendicular to $\vec{B}$ where the orbits are circular trajectories. Thus the motion follows a helical trajectory with uniform velocity along the direction of the magnetic field, as depicted in Figure 2a.

Dirac monopole fields. In the background of the field $\vec{B}=\vec{B}_{g}$ of a single Dirac monopole (2.9), the classical dynamics is also integrable. The integrals of motion are provided by the Poincare vector $\vec{K}$, which is the sum of the orbital angular momentum $\vec{L}$ with the angular momentum of the electromagnetic field due to the electric charge and the Dirac monopole. The conservation of the Poincare vector confines the motion of the electron to the surface of a cone, with apex at the location of the monopole, which precesses around the direction $\vec{K}$ with a time-dependent angular frequency 


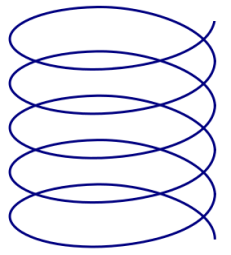

(a)

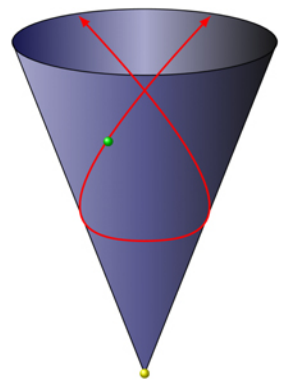

(b)

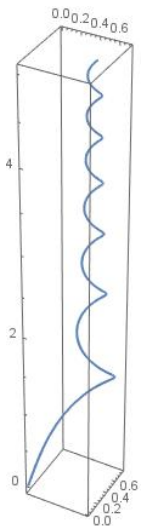

(c)

Figure 2: Classical electron trajectories in the background of: (a) a uniform magnetic field; (b) a Dirac monopole field; and (c) an axial magnetic field.

as depicted in Figure 2b. In this case the electric charge never reaches the magnetic monopole, so the motion takes place in $M^{\times}$and nonassociativity of the magnetic Poisson brackets plays no role [72]. This dynamical system was generalized recently to the case of multiple electric charges and monopoles in [73], where it was shown that the Jacobiator of the corresponding magnetic Poisson brackets vanishes only if all charges have identical ratio of electric to magnetic charge, or if an electron never collides with a monopole.

Axial magnetic fields. In the case of a uniform distribution of magnetic charge $\rho$, the closest analog to the case of a uniform magnetic field considered above is when the magnetic field is aligned along the direction of a fixed unit vector $\vec{n}$ in $\mathbb{R}^{3}$, so that $\vec{B}=\vec{B}_{\rho, \vec{n}}$ with

$$
\vec{B}_{\rho, \vec{n}}=\rho(\vec{x} \cdot \vec{n}) \vec{n}
$$

The corresponding Lorentz force equation was studied in [74], where it was shown that the motion is again completely integrable. Again the motion is uniform along the direction $\vec{n}$ of the magnetic field, and in the plane perpendicular to $\vec{n}$ the equations of motion are those of a harmonic oscillator with a time-dependent angular frequency, which can be solved in terms of Fresnal integrals. In this case the trajectories follow an Euler spiral with uniform velocity along the direction $\vec{n}$ of the magnetic field, as depicted in Figure 2c.

Rotationally symmetric magnetic fields. The situation is drastically different in the case of a uniform distribution of magnetic charge $\rho$ which sources the rotationally symmetric magnetic field $\vec{B}=\vec{B}_{\rho, \text { rot }}$ with

$$
\vec{B}_{\rho, \mathrm{rot}}=\frac{1}{3} \rho \vec{x}
$$

In [72] it was shown that the Lorentz force equation can be transformed via a time reparameterization of the trajectories into the equation of motion of a dissipative system, which governs the motion of an electric charge in the field of a Dirac monopole $\vec{B}_{g}$ with an additional time-dependent 
frictional force accounting for the background magnetic charge $\rho$. In this case, the motion is no longer confined in any direction, and the dynamics is not integrable. We shall discuss another interpretation of this dissipative nature of the dynamics later on.

We shall now turn to the quantization of the dynamics and ask, in the case of distributions of magnetic charge, if there is a sensible version of nonassociative quantum mechanics accompanying the classical analysis above.

\subsection{Quantization of magnetic Poisson brackets}

Let us examine the general problem of quantizing the magnetic Poisson brackets (2.3) on $M=\mathbb{R}^{d}$. Quantization can be generally regarded as a one-to-one linear map $f \mapsto \mathscr{O}_{f}$ from functions $f \in C^{\infty}(\mathscr{M})$ to symbols $\mathscr{O}_{f}$ which admit some binary composition operation $\circ$ with unit; the precise specification of the operators $\mathscr{O}_{f}$ is intentionally left vague for the moment, as this will depend crucially on the quantization scheme chosen. The map $\mathscr{O}$ should satisfy the algebraic property

$$
\lim _{\hbar \rightarrow 0} \frac{1}{2} \mathscr{O}^{-1}\left(\mathscr{O}_{f} \circ \mathscr{O}_{g}+\mathscr{O}_{g} \circ \mathscr{O}_{f}\right)=f g
$$

where $f g$ is the usual pointwise multiplication of phase space functions $f, g \in C^{\infty}(\mathscr{M})$, together with the generalized Bohr correspondence principle

$$
\lim _{\hbar \rightarrow 0} \frac{\mathrm{i}}{\hbar} \mathscr{O}^{-1}\left(\left[\mathscr{O}_{f}, \mathscr{O}_{g}\right]_{\circ}\right)=\{f, g\}_{B},
$$

where $\left[\mathscr{O}_{f}, \mathscr{O}_{g}\right]_{\circ}=\mathscr{O}_{f} \circ \mathscr{O}_{g}-\mathscr{O}_{g} \circ \mathscr{O}_{f}$. This is a generalization of Dirac's quantization framework which deforms the algebraic and geometric structures of the classical phase space $\mathscr{M}$ in terms of some parameter $\hbar$, physically understood as Planck's constant. As usual, the quantization map does not generally preserve the classical magnetic Poisson brackets. However, it does represent the brackets (2.4) of local coordinates as the quantum commutators

$$
\left[\mathscr{O}_{x^{i}}, \mathscr{O}_{x^{j}}\right]_{\circ}=0, \quad\left[\mathscr{O}_{x^{i}}, \mathscr{O}_{p_{j}}\right]_{\circ}=\mathrm{i} \hbar \delta^{i}{ }_{j} \quad \text { and } \quad\left[\mathscr{O}_{p_{i}}, \mathscr{O}_{p_{j}}\right]_{\circ}=-\mathrm{i} \hbar B_{i j}\left(\mathscr{O}_{x}\right)
$$

To study the representation of the quantum kinematics, it is useful to introduce, for any $\hbar \neq 0$, the magnetic translation operators

$$
\mathscr{P}_{v}=\exp _{\circ}\left(\frac{\mathrm{i}}{\hbar} \mathscr{O}_{p \cdot v}\right)
$$

where the operation $\exp _{\circ}$ denotes the usual formal power series expansion of the exponential function with powers taken with the composition $\circ$. By the commutation relations (2.19), they formally implement translations on configuration space by constant vectors $v \in \mathbb{R}^{d}$ at the quantum level:

$$
\mathscr{P}_{v}^{-1} \circ \mathscr{O}_{x^{i}} \circ \mathscr{P}_{v}=\mathscr{O}_{x^{i}+v^{i}}
$$

where the inverse is taken with respect to $\circ$. As noticed originally by [46], the operators $\mathscr{P}_{v}$ do not represent the translation group $\mathbb{R}^{d}$, as expected because the background 2-form field $B$ breaks the translational symmetries of the dynamical system on $\mathbb{R}^{d}$. Instead, by formally iterating the 
conjugation action (2.21) using (2.19), one finds that they satisfy the composition and association relations

$$
\begin{gathered}
\mathscr{P}_{w} \circ \mathscr{P}_{v}=\Pi_{v, w}(x) \mathscr{P}_{v+w}, \\
\mathscr{P}_{w} \circ\left(\mathscr{P}_{v} \circ \mathscr{P}_{u}\right)=\Omega_{u, v, w}(x)\left(\mathscr{P}_{w} \circ \mathscr{P}_{v}\right) \circ \mathscr{P}_{u},
\end{gathered}
$$

where

$$
\Pi_{v, w}(x)=\exp \left(-\frac{\mathrm{i}}{\hbar} \int_{\triangle^{2}(x ; v, w)} B\right)
$$

is a phase factor determined by the magnetic flux through the triangle $\triangle^{2}(x ; v, w)$ based at $x$ and spanned by the translation vectors $v, w$, while

$$
\Omega_{u, v, w}(x)=\exp \left(\frac{\mathrm{i}}{\hbar} \int_{\triangle^{3}(x ; u, v, w)} H\right)
$$

is a phase factor determined by the total magnetic charge enclosed by the tetrahedron $\triangle^{3}(x ; u, v, w)$ based at $x$ and spanned by the vectors $u, v, w$ in $\mathbb{R}^{d}$, or equivalently by Stokes' theorem, the total flux through the faces of the tetrahedron. In general, $\Pi_{v, w}(x)$ defines a 2-cochain of the translation group $\mathbb{R}^{d}$ with coboundary the 3-cocycle $\Omega_{u, v, w}(x)$ in the group cohomology of $\mathbb{R}^{d}$ with values in $C^{\infty}(M, U(1))$. From this point of view, defining a quantization map means providing a representation, in a suitable sense, of the algebra (2.22) of magnetic translation operators.

Let us now explicitly confirm these formal calculations in our specific cases of interest.

Maxwell theory. In the $d$-dimensional generalization of the classical Maxwell theory, the magnetic field $B$ on $M=\mathbb{R}^{d}$ is a closed 2-form, $\mathrm{d} B=0$, so there exists a globally defined 1-form $A$ such that $B=\mathrm{d} A$. The quantum theory can then be described via canonical quantization. The quantization map in this case takes functions on phase space $\mathscr{M}$ to operators acting on the quantum Hilbert space $\mathscr{H}=\mathrm{L}^{2}(M)$ of square-integrable functions on configuration space with respect to the usual Lebesgue measure $\mathrm{d} x$ on $\mathbb{R}^{d}$, with the composition operation $\circ$ given by the usual associative composition of linear operators. This constructs the Schrödinger representation of the algebra of magnetic translations where the position and kinematical momentum operators are represented as

$$
\left(\mathscr{O}_{x^{i}} \psi\right)(x)=x^{i} \psi(x) \quad \text { and } \quad\left(\mathscr{O}_{p_{i}} \psi\right)(x)=-\mathrm{i} \hbar \frac{\partial}{\partial x^{i}} \psi(x)+A_{i}(x) \psi(x)
$$

on wavefunctions $\psi \in \mathscr{H}$. The magnetic translation operators in this case can be represented by the Wilson lines

$$
\left(\mathscr{P}_{v} \psi\right)(x)=\exp \left(-\frac{\mathrm{i}}{\hbar} \int_{[x-v, x]} A\right) \psi(x-v)
$$

where $[x-v, x]$ is the straight line in $\mathbb{R}^{d}$ from the point $x-v$ to $x$. An explicit calculation verifies the composition relation in (2.22) in this case where, since $H=\mathrm{d} B=0$, the coboundary $\Omega_{u, v, w}(x)=1$ is trivial. Thus in this instance $\Pi_{v, w}(x)$ defines a 2-cocycle on $M=\mathbb{R}^{d}$ with values in $C^{\infty}(M, U(1))$, and the magnetic translation operators generate a weak projective representation of the translation group $\mathbb{R}^{d}$ on $\mathscr{H}$, the adjective 'weak' refering to the $x$-dependence of the 2-cocycle that modifies 
the usual cocycle condition by the action of $\mathbb{R}^{d}$ on $\Pi_{v, w}(x)$ (see e.g. [75]). In the case where the components of the 2-form $B$ are constant, the 2-cocycle simplifies to

$$
\Pi_{v, w}=\mathrm{e}^{-\frac{\mathrm{i}}{2 \hbar} B(v, w)}
$$

independently of $x$, and we obtain the standard projective representation of translations in the background of a constant magnetic field.

The magnetic translation operators also provide the general form of the quantization map from generic phase space functions $f \in C^{\infty}(\mathscr{M})$, which are integrable with respect to the Lebesgue measure $\mathrm{d} X$ on $\mathscr{M}$, to trace-class operators $\mathscr{O}_{f}$ on the Hilbert space $\mathscr{H}$. This is called the magnetic Weyl correspondence, and it is determined by a family of operators $\Delta_{\mathscr{O}}(X)$ on $\mathscr{H}$ parameterized by points $X=(x, p)$ of $\mathscr{M}$ which are defined on wavefunctions $\psi \in \mathscr{H}$ by

$$
\left(\Delta_{\mathscr{O}}(x, p) \psi\right)(y)=\mathrm{e}^{\frac{\mathrm{i} \hbar}{2} p \cdot x} \mathrm{e}^{-\mathrm{i} p \cdot y}\left(\mathscr{P}_{x} \psi\right)(y) .
$$

From (2.22) it follows that this operator satisfies

$$
\Delta_{\mathscr{O}}(X)^{\dagger}=\Delta_{\mathscr{O}}(-X) .
$$

Then the magnetic Weyl correspondence defines operators $\mathscr{O}_{f}$ given by

$$
\mathscr{O}_{f}=\int_{\mathscr{M}}\left(\int_{\mathscr{M}} \mathrm{e}^{\mathrm{i} \omega_{0}(X, Y)} f(Y) \frac{\mathrm{d} Y}{(2 \pi)^{d}}\right) \Delta_{\mathscr{O}}(X) \frac{\mathrm{d} X}{(2 \pi)^{d}}
$$

for suitable class functions $f \in C^{\infty}(\mathscr{M})$, where $\omega_{0}$ is the Darboux 2-form (2.1). The magnetic Weyl correspondence $f \mapsto \mathscr{O}_{f}$ is unitary,

$$
\mathscr{O}_{f(X)}^{\dagger}=\mathscr{O}_{\overline{f(-X)}},
$$

and it is invertible as a consequence of completeness of the Hilbert space $\mathscr{H}$. Its inverse can be used to pull back the product of two operators $\mathscr{O}_{f}, \mathscr{O}_{g}$ to a noncommutative product of the corresponding phase space functions $f, g$. This defines the associative magnetic Moyal-Weyl star product through $\mathscr{O}_{f \star_{B} g}=\mathscr{O}_{f} \mathscr{O}_{g}$, which when $B$ is constant is given explicitly by the twisted convolution product

$$
\left(f \star_{B} g\right)(X)=\frac{1}{(\pi \hbar)^{d}} \int_{\mathscr{M}} \int_{\mathscr{M}} \mathrm{e}^{-\frac{2 \mathrm{i}}{\hbar} \omega_{B}(Y, Z)} f(X-Y) g(X-Z) \mathrm{d} Y \mathrm{~d} Z,
$$

where $\omega_{B}$ is the twisted symplectic 2-form (2.2); see e.g. [75] for the explicit expression in the general case. This generalizes the standard Moyal-Weyl star product on canonical phase space for $B=0$, while for any 2 -form $B$ it recovers the ordinary convolution product between integrable functions when $\hbar=0$.

This shows that the magnetic translation operators also provide a bridge between the canonical formulation of quantum mechanics and the somewhat less known phase space formulation of quantum mechanics, which is historically the origin of deformation quantization and star products, see e.g. [76]. In the phase space quantization scheme, the operators are the functions $f$ themselves, with the composition operation $\circ$ given by the star product $\star_{B}$. The deformation from classical to quantum observables is then characterized by the noncommutative and associative magnetic 
Moyal-Weyl star product of functions on phase space, which substitutes the pointwise product and contains the necessary information about the corresponding quantum system. In particular, the star product deforms the magnetic Poisson brackets in such a way as to contain all information related to the commutator of quantum operators $\mathscr{O}_{f}$. All of canonical quantum mechanics can be rephrased in the language of phase space quantum mechanics, with some caveats, through a dictionary which for later use we briefly summarise as follows:

- Wavefunctions are given by arbitrary complex-valued functions on phase space $\mathscr{M}$, while observables are real functions.

- The noncommutative composition of operators becomes the noncommutative star product of functions.

- Traces of operators become integrals of functions over $\mathscr{M}$.

- There is a state function $\Sigma \geqslant 0$ on $\mathscr{M}$ which is normalized, $\int_{\mathscr{M}} \Sigma(X) \mathrm{d} X=1$, and which plays the role of a density matrix determining the correlations of the quantum system. The state function originates through the inverse of the magnetic Weyl correspondence $f \mapsto \mathscr{O}_{f}$ in (2.30) taking quantum operators on $\mathscr{H}$ to functions on phase space, which determines the magnetic Wigner functions representing the density matrices that are quasi-probability distributions on $\mathscr{M}$. They heuristically give the quantization of the constant energy surfaces in phase space through the star product. Expectation values of operators $\mathscr{O}_{f}$ in the state $\Sigma$ are given by

$$
\left\langle\mathscr{O}_{f}\right\rangle_{\Sigma}=\int_{\mathscr{M}}\left(f \star_{B} \Sigma\right)(X) \mathrm{d} X
$$

Dirac monopoles. Quantization in the case when $H=\mathrm{d} B \neq 0$ is not straightforward, because the operator/state formulation of canonical quantum mechanics cannot handle nonassociative magnetic Poisson brackets: Operators acting on a separable Hilbert space always associate. The exception is the case of the Dirac monopole field (2.9). Recall that in this case the magnetic Poisson brackets are associative away from location of the monopole at the origin, i.e. on $M^{\times}=\mathbb{R}^{3} \backslash\{\overrightarrow{0}\}$. Recall also from the analysis of Section 2.3 that the electric charge in this background follows a classical trajectory that never reaches the monopole. In the quantum theory this means that the wavefunction of the electric charge should vanish at the origin $\overrightarrow{0}$, so that the quantum mechanics is also confined to $M^{\times}$.

To understand how to quantize the dynamics on $M^{\times}$, we observe that the quantization of Maxwell theory discussed above can be recast in a more geometric way: The globally defined 1form potential $A$ can be thought of as a connection on a (necessarily trivial) line bundle $L=M \times \mathbb{C}$ on $M$, with field strength the magnetic field $B=\mathrm{d} A$. Then the quantum Hilbert space of states can be thought of as the space $\mathscr{H}=\mathrm{L}^{2}(M, L)$ of square-integrable (global) sections of this line bundle, the Schrödinger representation of the kinematical momentum operators from (2.25) can be written in terms of covariant derivatives $D_{A}$ as $\mathscr{O}_{p_{i}} \psi=\left(D_{A}\right)_{i} \psi$ for $\psi \in \mathscr{H}$, and the magnetic translation operators (2.26) define parallel transport in $L$. This is just a reformulation of the canonical quantization problem as geometric quantization, which is of course superfluous on $M=\mathbb{R}^{d}$. 
However, this has the virtue of generalizing to non-trivial configuration spaces such as $M^{\times}=$ $\mathbb{R}^{3} \backslash\{\overrightarrow{0}\}$, which is topologically a 2-sphere $S^{2}$. The 2-form corresponding to the Dirac monopole field can be expressed from (2.10) as $B_{g}=\mathrm{d} A_{g, \vec{n}}$, where the locally defined 1-form $A_{g, \vec{n}}$ defines a non-trivial connection on a line bundle $L \rightarrow M^{\times}$, and the quantum Hilbert space is again the space of square-integrable (local) sections $\mathscr{H}=\mathrm{L}^{2}\left(M^{\times}, L\right)$. Line bundles on a 2-sphere can be non-trivial with a non-zero Chern number $n \in \mathbb{Z}$ that is obtained by integrating the field strength over $S^{2}$. Integrating the 2 -form $B_{g}$ over $S^{2}$ gives $2 \mathrm{eg} / \hbar$, which is hence the field strength of a line bundle $L$ if and only if

$$
n=\frac{2 e g}{\hbar} \in \mathbb{Z} .
$$

This is the celebrated Dirac quantization of electric charge, formulated geometrically in this way originally by [77]. The quantization condition (2.34) in turn implies that the 3 -cocycle $\Omega_{u, v, w}=1$ in (2.22) is trivial, and hence that the corresponding magnetic translation operators, defined by parallel transport in the non-trivial line bundle $L$, associate, consistently with their representability on the Hilbert space $\mathscr{H}$. Turning this argument around, the associativity of magnetic translations is equivalent to $\Omega_{u, v, w}=1$, which implies the Dirac quantization condition (2.34), formulated algebraically in this way originally by [46] (see also [78]). The quantization map $f \mapsto \mathscr{O}_{f}$ in this case can be constructed via a suitable magnetic Weyl correspondence $\Delta_{\mathscr{O}}(X)$ on the phase space $\mathscr{M}^{\times}$ of the configuration space $M^{\times}$, which induces an associative phase space star product constructed explicitly by $[79,80]$.

\subsection{Nonassociative quantum mechanics}

For generic smooth distributions of magnetic charge $H \in \Omega^{3}(M)$, standard canonical quantization breaks down. However, as first observed by [55], the magnetic Poisson brackets in these instances can still be quantized by appealing to the formalism of deformation quantization. For any smooth 3-form $H=\mathrm{d} B \in \Omega^{3}(M)$, Kontsevich formality provides a noncommutative and nonassociative star product on functions in $C^{\infty}(\mathscr{M})$ given as a formal power series in $\hbar$; we refer to the original treatment of [55] and to the review [65] for details of the Kontsevich expansion. For generic $H$, the Kontsevich formula can only be written as a formal power series expansion and computed order by order in the deformation parameter $\hbar$, which becomes increasingly complicated as the order increases.

When the components of $H$ are constant, we can choose the magnetic field to have components

$$
B_{i j}(x)=\frac{1}{3} H_{i j k} x^{k},
$$

which is the generalization of the rotationally symmetric magnetic field (2.16) in three dimensions. In this case the Kontsevich series can be summed explicitly to give a closed formal expression for the nonassociative star product in terms of the bidifferential operator determined by the magnetic Poisson bivector $\theta_{B}$ from Section 2.1 [55]. In [81] it was shown that this star product can be written in the form of a twisted convolution product which gives a convergent expression (as opposed to an asymptotic series) and reads as

$$
\left(f \star_{H} g\right)(X)=\frac{1}{(\pi \hbar)^{d}} \int_{\mathscr{M}} \int_{\mathscr{M}} \mathrm{e}^{-\frac{2 \mathrm{i}}{\hbar} \omega_{B}(Y, Z)} f(X-Y) g(X-Z) \mathrm{d} Y \mathrm{~d} Z,
$$


for suitable class functions $f, g \in C^{\infty}(\mathscr{M})$. Note that this is formally identical to the star product (2.32) which is written for constant $B$, with the difference here that the star product (2.36) is nonassociative, with the nonassociativity controlled by the constant magnetic charge $H$. This star product was subsequently computed by a variety of other means, see e.g. [72, 82].

In this framework, the quantum operators $\mathscr{O}_{f}$ are the functions $f \in C^{\infty}(\mathscr{M})$ themselves, and the composition operation $\circ$ is the nonassociative star product $\star_{H}$. The nonassociative magnetic translation operators in this case are given by the phase space functions

$$
\mathscr{P}_{v}:=\mathrm{e}^{\frac{\mathrm{i}}{\hbar} p \cdot v} .
$$

They satisfy the algebraic relations (2.22) with

$$
\begin{aligned}
\mathscr{P}_{w} \star_{H} \mathscr{P}_{v} & =\Pi_{v, w}(x) \mathscr{P}_{v+w}, \\
\mathscr{P}_{w} \star_{H}\left(\mathscr{P}_{v} \star_{H} \mathscr{P}_{u}\right) & =\Omega_{u, v, w}\left(\mathscr{P}_{w} \star_{H} \mathscr{P}_{v}\right) \star_{H} \mathscr{P}_{u},
\end{aligned}
$$

where

$$
\Pi_{v, w}(x)=\mathrm{e}^{-\frac{\mathrm{i}}{6 \hbar} H(x, v, w)} \quad \text { and } \quad \Omega_{u, v, w}=\mathrm{e}^{\frac{\mathrm{i}}{6 \hbar} H(u, v, w)}
$$

define a 3-cocycle of the translation group $\mathbb{R}^{d}$.

Although there is no standard canonical quantization framework available in this case, one can now adapt the formalism of phase space quantization outlined in Section 2.4 using the nonassociative star product. In [81] it is shown that the phase space formulation of nonassociative quantum mechanics obtained in this way is physically sensible: It passes all preliminary tests for a sensible quantum theory, such as reality, positivity and completeness of eigenstates of physical observables (real functions). It is a completely quantitative framework which gives novel predictions, such as modified uncertainty relations. See [71] for a discussion of some of the interesting predictions for the physics of electron propagation in fields of magnetic monopoles; we shall give a concrete application later on to explain some of the unusual physics in locally non-geometric backgrounds of string theory. This nonassociative quantum theory is in no conflict with the studies of Jordanian quantum mechanics discussed in Section 1.3, because the algebra of functions on phase space with the nonassociative star product (2.36) is not an alternative algebra: This was shown in [83] for a class of nonassociative star products which includes (2.36), and subsequently proven to be a general feature of nonassociative deformation quantization by [84]. At leading semi-classical order, the nonassociative algebra of functions is alternative, and this may be used to study a noncommutative Jordan algebra whose quantum moments can be developed perturbatively in $H$ [85]. This formalism was subsequently used to develop certain testable features of nonassociative quantum mechanics in $[86,87]$. In particular, the possibility of producing magnetic monopoles in particle colliders has been considered extensively, see e.g. [88] for a recent discussion and further references. In this way the present treatment of the physics of smooth distributions of magnetic monopoles may be of use to develop measurable predictions of nonassociativity, such as in the MoEDAL experiment at the Large Hadron Collider (LHC) which aims to pursue the quest for magnetic monopoles and dyons at LHC energies [89,90].

One undesirable feature of this deformation quantization approach, aside from the usual technical and conceptual issues arising in conventional phase space quantum mechanics [76], is that for 
magnetic charges beyond the constant case, the star product can only be developed as an asymptotic series in Planck's constant $\hbar$. From this point of view deformation quantization is not a true quantization in the physical sense, where Planck's constant can be set to some fixed finite value. It would therefore still be desirable to have an alternative form of canonical quantum mechanics available, in order to pursue better the foundations of this nonassociative quantum theory, and to seek further testable aspects of the model. What is missing in the theory is a nonassociative ana$\log$ of the magnetic Weyl correspondence $\Delta_{\mathscr{O}}(X)$, taking phase space functions to operators on a "Hilbert space". We conclude this section by briefly summarising three such attempts at a Hilbert space formulation of nonassociative quantum mechanics.

Symplectic realization. Symplectic realization is a well-known method from Poisson geometry that enables one to set up the problem of quantizing generic Poisson brackets in the realm of geometric quantization of phase spaces. In [74] it was demonstrated how to extend the technique to the case of magnetic Poisson brackets. The idea is to "double" the phase space $\mathscr{M}$ to an extended phase space with coordinates $\left(x^{i}, \tilde{x}^{i}, p_{i}, \tilde{p}_{i}\right)$ and the symplectic brackets

$$
\begin{aligned}
& \left\{x^{i}, p_{j}\right\}=\left\{\tilde{x}^{i}, p_{j}\right\}=\left\{x^{i}, \tilde{p}_{j}\right\}=\delta^{i}{ }_{j}, \\
& \left\{p_{i}, p_{j}\right\}=B_{i j}(x)+\frac{1}{2} \tilde{x}^{k}\left(\partial_{k} B_{i j}(x)-H_{i j k}(x)\right), \\
& \left\{p_{i}, \tilde{p}_{j}\right\}=\left\{\tilde{p}_{i}, p_{j}\right\}=\frac{1}{2} B_{i j}(x) .
\end{aligned}
$$

These brackets define an associative algebra and are inverse to a symplectic 2-form which pulls back to the twisted 2-form $\omega_{B}$ under the map which embeds the original phase space $\mathscr{M}$ into the extended phase space, or more concretely which restricts to $\omega_{B}$ by eliminating the auxiliary coordinates $\left(\tilde{x}^{i}, \tilde{p}_{i}\right)$. Some mathematical aspects of this symplectic realization are reviewed in [66].

On the extended phase space one can then introduce the $O(d, d) \times O(d, d)$-invariant Hamiltonian

$$
\widehat{\mathrm{H}}=\frac{1}{m} p_{I} \eta^{I J} p_{J}
$$

where $p_{I}=\left(p_{i}, \tilde{p}_{i}\right)$ and

$$
\eta=\left(\begin{array}{ll}
0 & 1 \\
1 & 0
\end{array}\right)
$$

is the $O(d, d)$-invariant metric. The corresponding Hamilton equations of motion for $d=3$ with the brackets (2.40) then reproduce the Lorentz force law (2.11) and (2.12) for the physical degrees of freedom $\left(x^{i}, p_{i}\right)$. This mimics the situation in double field theory [91], with one crucial difference. A consistent Hamiltonian reduction of the auxiliary degrees of freedom, via the imposition of suitable first or second class constraints, is possible if and only if there is no magnetic charge, $H=0$ : There is no polarisation of the extended symplectic algebra which is consistent with both the Lorentz force law and the nonassociative magnetic Poisson brackets [74]. This feature is seemingly related to the dissipative nature of the classical dynamics in a rotationally symmetric magnetic field that we discussed in Section 2.3, since a proper Hamiltonian formulation of dissipative systems also requires the introduction of auxiliary variables, representing a reservoir, in order to conserve the 
total energy. However, in the present case the total energy is already conserved, so the meaning of this analogy is not entirely clear. Thus while the formalism of symplectic realisation formally solves the problem of a Hilbert space formulation of the nonassociative quantum mechanics, the meaning of the auxiliary degrees of freedom, which cannot be eliminated, remains unclear. See the contribution of V. Kupriyanov to these proceedings for further details of this method.

2-Hilbert spaces. Nonassociativity implies the appearence of higher structures in algebra, where in addition to non-vanishing binary commutators of a multiplication one encounters non-zero ternary Jacobiators (or associators). These structures are natural from the point of view of the theory of monoidal categories, wherein one works with algebra objects not in the usual category of vector spaces, where associativity of tensor products holds on the nose, but in a category where associativity holds only up to a natural isomorphism called the associator. From the perspective of geometric quantization, this corresponds to the passage from line bundles on $M$, with 2-form field strength $B=\mathrm{d} A$ satisfying the Bianchi identity $\mathrm{d} B=0$, to gerbes on $M$ whose field strength is controlled by a closed 3 -form $H=\mathrm{d} B \neq 0$. In this case the usual quantum Hilbert space of sections of a line bundle should be replaced by an analog of sections of a gerbe, that has the structure of a 'categorified' Hilbert space called a 2-Hilbert space which is a certain monoidal category. Geometric quantization of the magnetic Poisson brackets for any magnetic field $B$ was worked out in this setting by [75], where the 3-cocycles $\Omega_{u, v, w}(x)$ appearing in (2.22) are interpreted as controlling suitable higher versions of a (weak) projective representation, implemented by magnetic translation functors $\mathscr{P}_{v}$ on the 2-Hilbert space; this utilizes a higher notion of parallel transport in a gerbe. While again this approach formally captures a Hilbert space formulation of the nonassociative quantum mechanics, the physical meaning and development of the higher structures involved are at present unclear. A concise review of this approach can be found in [66].

Transgression to loop space. In higher geometry it is well-known that the nonassociativity features of a gerbe on $M$ can be traded for more conventional noncommutative features of a line bundle on the loop space $C^{\infty}\left(S^{1}, M\right)$ of embeddings of a circle $S^{1}$ into the configuration space $M$; this mapping is called transgression and it has a natural interpretation of trading particle degrees of freedom for closed string degrees of freedom. The field strength of a gerbe on $M$ is sent to the field strength of a line bundle on the closed string configuration space $C^{\infty}\left(S^{1}, M\right)$ under the transgression map, and on the latter one can proceed to apply the standard techniques of geometric quantization; this approach was pursued in [40,41]. However, this approach simply hides the higher structure of the 2-Hilbert space of sections of a gerbe in a Hilbert space of sections of a line bundle over an infinitedimensional configuration space of closed strings, again making explicit constructions and their physical interpretations unwieldy. Once more, although the problem of a Hilbert space approach to quantization is formally solved, many technical and conceptual difficulties remain awaiting for further exploration. The discussion of this paragraph motivates considerations of these higher nonassociativity structures in terms of closed strings, to which we now turn.

\section{Closed strings in locally non-geometric flux backgrounds}

\subsection{The $R$-flux model}

We now turn to our attention to somewhat more conjectural applications to non-geometric 
string theory. At the algebraic level, the pertinent phase space brackets are obtained by applying a certain'duality' transformation to the magnetic Poisson brackets from Section 2.1. For this, we invoke Born reciprocity, which interchanges the roles of local position and momentum coordinates through the mapping $(x, p) \mapsto(p,-x)$ on phase space $\mathscr{M}$ of order 4 . This map preserves the canonical symplectic 2-form $\omega_{0}$ on $\mathscr{M}$, but it does not preserve the twisted 2-form $\omega_{B}$, and so is not a natural symmetry of the magnetic Poisson brackets. Instead, it has a natural geometric interpretation by regarding the phase space $\mathscr{M}=T^{*} M$ as a para-Hermitian manifold [92] where it corresponds to an $O(d, d)$-transformation which preserves the Lorentzian metric (2.42) and takes the magnetic Poisson brackets (2.4) of local coordinates, depending on a 2-form $B \in \Omega^{2}(M)$ on configuration space $M$, to the twisted Poisson brackets

$$
\left\{x^{i}, x^{j}\right\}_{\beta}=-\beta^{i j}(p), \quad\left\{x^{i}, p_{j}\right\}_{\beta}=\delta^{i}{ }_{j} \quad \text { and } \quad\left\{p_{i}, p_{j}\right\}_{\beta}=0,
$$

depending on a 2-form $\beta \in \Omega^{2}\left(M^{*}\right)$ on the dual momentum space $M^{*}=\mathbb{R}^{d}$. In this case the twisting is by a 3 -form on momentum space $M^{*}$, called the ' $R$-flux' $\ell_{s}^{3} R=\mathrm{d} \beta \in \Omega^{3}\left(M^{*}\right)$, where $\ell_{s}$ is the string length. This now gives a nonassociative configuration space with the nonvanishing coordinate Jacobiators

$$
\left\{x^{i}, x^{j}, x^{k}\right\}_{\beta}=-\ell_{s}^{3} R^{i j k}(p) .
$$

These brackets have a natural formulation as a 2-term $L_{\infty}$-algebra which was developed in [55,93].

The dynamical system captured by these dual brackets is called the $R$-flux model, and it conjecturally describes the phase space of closed strings propagating in 'locally non-geometric' $R$-flux backgrounds of string theory, which have no formulation as a conventional spacetime [53,55]. As for the case of the magnetic Poisson brackets, the prototypical example arises in $d=3$ spacetime dimensions with constant $R$-flux. This originates from a type II background containing a 3-torus $M=T^{3}$ with constant $H$-flux (proportional to the volume form) through a chain of successive Tdualities which gives rise to geometric and non-geometric fluxes that are depicted schematically as [94]

$$
H_{i j k} \stackrel{\mathrm{T}_{i}}{\longleftrightarrow} f_{j k}^{i} \stackrel{\mathrm{T}_{j}}{\longleftrightarrow} Q^{i j}{ }_{k} \stackrel{\mathrm{T}_{k}}{\longleftrightarrow} R^{i j k}
$$

where $\mathrm{T}_{i}$ denotes a $\mathrm{T}$-duality transformation along the $i$-th coordinate direction which sends string winding numbers $\left(w^{i}\right) \in H_{1}\left(T^{3}, \mathbb{Z}\right)=\mathbb{Z} \oplus \mathbb{Z} \oplus \mathbb{Z}$ to momentum modes $\left(p_{i}\right)$ (and vice-versa). Starting from the geometric background $\left(T^{3}, H\right)$, a T-duality takes this to the Heisenberg nilmanifold $\tilde{T}^{3}$, which is a geometric background with no $B$-field and a metric flux (torsion) $f$. Applying another T-duality then results in a T-fold [95], a non-geometric background which is not globally well-defined with the transition functions controlled by a ' $Q$-flux'. A final T-duality then results in a background which cannot be described even locally in conventional geometric terms, characterised by an $R$-flux. The triple T-duality transformation $\mathrm{T}_{i j k}$ which sends the $H$-flux

$$
H_{i j k}=\partial_{[i} B_{j k]}
$$

to the $R$-flux

$$
R^{i j k}=\hat{\partial}^{[i} \beta^{j k]}
$$


is best understood in double field theory [96], where it maps the 2-form NS-NS $B$-field to a bivector $\beta$ with $\hat{\partial}^{i}$ denoting 'modified derivatives' with respect to the coordinates dual to the string winding modes. This is completely analogous to the $O(d, d)$-transformation applied to the magnetic Poisson brackets above.

In the quantum theory, a suitable substitute for canonical quantization of locally non-geometric closed strings is provided by the deformation quantization of Section 2.5, with the replacements of phase space coordinates $X^{I}=\left(p_{i},-x^{i}\right)$ and the magnetic field $B$ with $\beta$ everywhere; in the case of a constant $R$-flux, we denote the corresponding nonassociative star product obtained from (2.36) with these substitutions by $\star_{R}$. The phase space formulation of nonassociative quantum mechanics discussed in Section 2.5 can then be used to quantitatively explore the physical implications of the local non-geometry of the configuration space [81]. As an application, let us compute the expectation values of the oriented volume uncertainty operators

$$
V^{i j k}=\left\langle\frac{1}{2}\left[\Delta x^{i}, \Delta x^{j}, \Delta x^{k}\right]_{\star_{R}}\right\rangle_{\Sigma}=\int_{\mathscr{M}}\left(\frac{1}{2}\left[\Delta x^{i}, \Delta x^{j}, \Delta x^{k}\right]_{\star_{R}} \star_{R} \Sigma\right)(X) \mathrm{d} X
$$

determined by the Jacobiators of the position uncertainty operators $\Delta x^{i}=x^{i}-\left\langle x^{i}\right\rangle_{\Sigma}$ for an arbitrary state function $\Sigma$. This quantity computes the quantum volume of the tetrahedron in configuration space spanned by the coordinate uncertainties in the given directions. While this quantity would obviously vanish in the associative case, in the present case it can be explicitly computed to give a non-zero quantum of volume [81]

$$
V^{i j k}=\frac{\hbar^{2} \ell_{s}^{3}}{2} R^{i j k}
$$

independently of the chosen state $\Sigma$. This corroborates the expectations that particles cannot be used as probes of the $R$-flux background [97]. This can be argued in $d=3$ dimensions via the Freed-Witten anomaly, which forbids D3-branes from wrapping a 3-torus $T^{3}$ with nonvanishing $H$-flux. Under a triple T-duality $\mathrm{T}_{i j k}, \mathrm{D} 3$-branes map to D0-branes and the $H$-flux to the $R$-flux, and hence D0-branes cannot propagate in locally non-geometric string backgrounds.

\subsection{How closed strings see nonassociativity}

The nonassociative phase space structure of the $R$-flux model raises some curiosities concerning basic string physics, insofar that at the level of the two-dimensional worldsheet conformal field theory one does not expect any such structures to arise (these would otherwise violate axioms of conformal field theory). The issue was clarified by [98] which demonstrated explicitly how to pass from nonassociative phase space star products to certain ternary products which capture the nonassociativity of configuration space fields in a way that is consistent with conformal invariance. For this, one defines configuration space triproducts in the $R$-flux model through

$$
\left(f_{1} \triangle f_{2} \Delta f_{3}\right)(x)=\left.\left(f_{1}(x) \star_{R} f_{2}(x)\right) \star_{R} f_{3}(x)\right|_{p=0}
$$

for any three functions $f_{1}, f_{2}, f_{3} \in C^{\infty}(M)$, where a particular bracketing of the nonassociative star product has been chosen. By Fourier transforming the configuration space fields $f_{i}$ on $M$ to fields $\tilde{f}_{i}$ on Fourier space $\tilde{M}$ one can express this triproduct as a twist of the usual Fourier convolution 
product of three fields as [98]

$$
\begin{aligned}
\left(f_{1}\right. & \left.\triangle f_{2} \Delta f_{3}\right)(x) \\
\quad & =\int_{\tilde{M}} \int_{\tilde{M}} \int_{\tilde{M}} \tilde{f}_{1}\left(k_{1}\right) \tilde{f}_{2}\left(k_{2}\right) \tilde{f}_{3}\left(k_{3}\right) \mathrm{e}^{-\frac{\mathrm{i} \hbar^{2} \ell_{3}^{3}}{4} R\left(k_{1}, k_{2}, k_{3}\right)} \mathrm{e}^{\mathrm{i}\left(k_{1}+k_{2}+k_{3}\right) \cdot x} \frac{\mathrm{d} k_{1}}{(2 \pi)^{d}} \frac{\mathrm{d} k_{2}}{(2 \pi)^{d}} \frac{\mathrm{d} k_{3}}{(2 \pi)^{d}} .
\end{aligned}
$$

One can introduce a natural 3-bracket via antisymmetrization of the triproducts to get

$$
\left[f_{1}, f_{2}, f_{3}\right]_{\triangle}=\frac{1}{3} \sum_{\sigma \in S_{3}}(-1)^{|\sigma|} f_{\sigma(1)} \Delta f_{\sigma(2)} \Delta f_{\sigma(3)}
$$

where the sum runs through all permutations of degree 3. This naturally quantizes the 3-bracket defined by the classical Jacobiator (3.2) of the $R$-flux model:

$$
\left[x^{i}, x^{j}, x^{k}\right]_{\triangle}=\hbar^{2} \ell_{s}^{3} R^{i j k} .
$$

In $d=3$ dimensions, the triproduct (3.9) was independently postulated by Takhtajan [10] over 20 years earlier as a candidate quantization of the canonical Nambu-Poisson 3-bracket (1.16) on $\mathbb{R}^{3}$.

These triproducts were rediscovered by [54] through calculations of the scattering of momentum states of closed string tachyon vertex operators

$$
V_{k}(z, \bar{z})=: \exp (\mathrm{i} k \cdot x(z, \bar{z})):
$$

in a linearisation of conformal field theory on flat space in the $R$-flux background. Computing the 3-point functions of such states then reproduces the triproduct to linear order in $R$, and indeed the all orders expression for the multiplication of vertex operators was conjectured to be given by the exact expression (3.9):

$$
\left\langle V_{k_{1}} V_{k_{2}} V_{k_{3}}\right\rangle_{R}=\exp \left(-\frac{\mathrm{i} \hbar^{2} \ell_{s}^{3}}{4} R\left(k_{1}, k_{2}, k_{3}\right)\right) .
$$

The precise meaning of this formula was thus subsequently clarified by [98]: The triproduct (3.9) on configuration space is inherited from the nonassociative star product on phase space. Both the star product and the triproduct violate the strong constraint of double field theory [57,99], which thus forbids a conventional spacetime interpretation, but on-shell associativity of conformal field theory amplitudes is retrieved as a result of the integral identity [98]

$$
\int_{M}\left(f_{1} \triangle f_{2} \triangle f_{3}\right)(x) \mathrm{d} x=\int_{M} f_{1}(x) f_{2}(x) f_{3}(x) \mathrm{d} x .
$$

Thus nonassociativity is an off-shell effect, invisible to the on-shell closed strings. See [65] for a detailed discussion of various other caveats associated with these and other derivations of closed string nonassociativity.

\subsection{Nonassociative gravity}

Given that the closed string sector contains gravity, it is natural to ask if there is a consistent nonassociative theory of gravity which governs the low-energy dynamics of closed strings in locally non-geometric backgrounds. This endeavour has been pursued at length by developing a theory of nonassociative (Riemannian) differential geometry through a generalization of the usual twist 
deformation techniques to the case where the twist is provided by a quasi-Hopf algebra 2-cochain, whose coboundary is a 3-cocycle controlling nonassociativity in a similar way as previously [81, 98,100-104]. Progress in this direction has been slow due to the extremely complicated nature of the technical constructions required.

The main result has been the development of a metric formulation of nonassociative gravity on the phase space of the $R$-flux model. One can construct a Ricci tensor as well as a unique metric-compatible torsion-free connection, playing the role of the Levi-Civita connection in ordinary Riemannian geometry [104]. Using a projection to configuration space analogous to that described in Section 3.2, this results in a non-trivial real deformation of the spacetime Ricci tensor given by [104]

$$
\begin{aligned}
\operatorname{Ric}_{i j}^{\circ}=\operatorname{Ric}_{i j}+\frac{\hbar^{2} \ell_{s}^{3}}{4} R^{a b c}\left(\partial_{k}\right. & \left(\partial_{a} g^{k l}\left(\partial_{b} g_{l m}\right) \partial_{c} \Gamma_{i j}^{m}\right)-\partial_{j}\left(\partial_{a} g^{k l}\left(\partial_{b} g_{l m}\right) \partial_{c} \Gamma_{i k}^{m}\right) \\
& +\partial_{c} g_{m n}\left(\partial_{a}\left(g^{l m} \Gamma_{l j}^{k}\right) \partial_{b} \Gamma_{i k}^{n}-\partial_{a}\left(g^{l m} \Gamma_{l k}^{k}\right) \partial_{b} \Gamma_{i j}^{n}\right. \\
& \left.\left.+\left(\Gamma_{i k}^{l} \partial_{a} g^{k m}-\partial_{a} \Gamma_{i k}^{l} g^{k m}\right) \partial_{b} \Gamma_{l j}^{n}-\left(\Gamma_{i j}^{l} \partial_{a} g^{k m}-\partial_{a} \Gamma_{i j}^{l} g^{k m}\right) \partial_{b} \Gamma_{l k}^{n}\right)\right)
\end{aligned}
$$

where $\operatorname{Ric}_{i j}$ is the Ricci tensor of a metric $g_{i j}$ on $M$ with Christoffel symbols $\Gamma_{i j}^{k}$. This expression holds to linear order in the $R$-flux, which is also the order at which the conformal field theory calculations of [54] are reliable. The fact that this quantity is real is a remarkable feature of the theory, as the Ricci tensor on phase space is complex-valued but its imaginary parts vanish under the projection to spacetime.

The final goal of nonassociative gravity is to mimic what happens in the well-known case of the open string sector with constant $B$-field. In that case the low-energy effective theory of D-branes wrapped on a $d$-dimensional torus $T^{d}$ is a supersymmetric noncommutative Yang-Mills theory which is invariant under open string $S O(d, d ; \mathbb{Z})$ T-duality. One then wishes to find an equivalent $O(d, d)$-invariant (off-shell) nonassociative version of the closed string effective action

$$
S=\frac{1}{16 \pi G} \int_{M} \sqrt{g}\left(\text { Ric }-\frac{1}{12} \mathrm{e}^{-\phi / 3} H_{i j k} H^{i j k}-\frac{1}{6} \partial_{i} \phi \partial^{i} \phi+\cdots\right) \mathrm{d} x,
$$

where $G$ is the gravitational constant, $\phi$ is the string dilaton field, and we have written only the bosonic part of the full type II supergravity action for brevity. Although much remains to be seen from such an effective theory, we can move on and seek the generalizations of these string theory backgrounds in their uplifts to M-theory.

\section{M2-branes in locally non-geometric flux backgrounds}

\subsection{M-theory lift of the $R$-flux model}

Type IIA string theory on a background containing the spacetime $M$ lifts to M-theory on the total space $M^{\prime}$ of a circle bundle

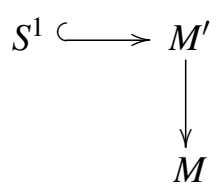


where the radius $\lambda$ of the circle fibres geometrizes the string coupling $g_{s}$. The lifts of the threedimensional constant $R$-flux backgrounds to locally non-geometric backgrounds of M-theory was considered for compactifications to four dimensions in [60] and to higher dimensions in [105]; we focus here on the former lift. One starts from a double T-duality taking the Heisenberg nilmanifold $M=\tilde{T}^{3}$ to the $R$-flux background:

$$
f_{j k}^{i} \stackrel{\mathrm{T}_{j k}}{\longrightarrow} R^{i j k}=R \varepsilon^{i j k} .
$$

For simplicity we consider the lift of this chain to M-theory on the trivial circle bundle $M^{\prime}=M \times S^{1}$. We denote the local coordinates on $M$ by $x^{i}, i=1,2,3$, as previously, and on the M-theory circle $S^{1}$ by $x^{4}$; we collect these coordinates into the four-dimensional coordinate vector $\vec{x}=\left(x^{\mu}\right)=\left(x^{i}, x^{4}\right)$ of $M^{\prime}$. In this case the T-duality $\mathrm{T}_{j k}$ lifts to a $\mathrm{U}$-duality $\mathrm{U}_{\mu v \rho}$ which sends membrane wrapping modes $\left(w^{i j}\right) \in H_{2}\left(M^{\prime}, \mathbb{Z}\right)$ to momentum modes $\left(p_{i}\right)$ (and vice-versa). The lift of the non-geometric string theory $R$-flux to M-theory can be described explicitly in $S L(5)$ exceptional field theory $[106,107]$. The U-duality $\mathrm{U}_{\mu \nu \rho}$ takes the 3-form $C$-field $C_{\mu \nu \rho}$ of 11-dimensional supergravity to a trivector $\Omega^{\mu v \rho}$ which is a potential for the M-theory $R$-flux

$$
R^{\mu, v \rho \alpha \beta}=\hat{\partial}^{\mu[v} \Omega^{\rho \alpha \beta]},
$$

where $\hat{\partial}^{\mu \nu}$ denotes a 'modified derivative' with respect to the coordinates conjugate to M2-brane wrapping modes; this is not a 5-vector but rather a mixed symmetry tensor whose first index transforms as a vector under four-dimensional rotations in $S O(4)$ and whose remaining four indices transform in the totally antisymmetric representation of $S O(4)$. The explicit choice of nonvanishing components

$$
R^{4, \mu v \alpha \beta}=R \varepsilon^{\mu v \alpha \beta}
$$

which we shall often make in what follows, then breaks the $S L(5)$ symmetry to $S O(4)$.

The quasi-Poisson brackets of the M2-brane phase space in the locally non-geometric $R$-flux background in this case were conjecturally described in [60]. Recall from Section 3.1 that there are no D0-branes permitted on the string background $M$ with $R$-flux. Since D0-brane charges in type IIA string theory lift to momentum modes along the M-theory circle, this implies that the momentum $p_{4}=0$ vanishes along the M-theory direction. We can write this constraint on the locally non-geometric M-theory background more covariantly in the form

$$
R^{\mu, v \rho \alpha \beta} p_{\mu}=0,
$$

which is trivially satisfied in the absence of M-theory $R$-flux, but otherwise cuts out a one-dimensional subspace of the phase space. In this case the membrane is purported to have a sevendimensional phase space $\mathscr{M}^{\prime}$ with quasi-Poisson brackets given by

$$
\begin{aligned}
& \left\{x^{i}, x^{j}\right\}_{\lambda}=\ell_{s}^{3} R^{4, i j k 4} p_{k} \quad \text { and } \quad\left\{x^{4}, x^{i}\right\}_{\lambda}=\lambda \ell_{s}^{3} R^{4,1234} p^{i} \\
& \left\{x^{i}, p_{j}\right\}_{\lambda}=\delta^{i}{ }_{j} x^{4}+\lambda \varepsilon^{i}{ }_{j k} x^{k} \quad \text { and } \quad\left\{x^{4}, p_{i}\right\}_{\lambda}=\lambda^{2} x_{i}, \\
& \left\{p_{i}, p_{j}\right\}_{\lambda}=-\lambda \varepsilon_{i j k} p^{k}
\end{aligned}
$$


which have the Jacobiators

$$
\begin{aligned}
& \left\{x^{i}, x^{j}, x^{k}\right\}_{\lambda}=\ell_{s}^{3} R^{4, i j k 4} x^{4} \quad \text { and } \quad\left\{x^{i}, x^{j}, x^{4}\right\}_{\lambda}=-\lambda^{2} \ell_{s}^{3} R^{4, i j k 4} x_{k}, \\
& \left\{p_{i}, x^{j}, x^{k}\right\}_{\lambda}=\lambda \ell_{s}^{3} R^{4,1234}\left(\delta_{i}{ }^{j} p^{k}-\delta_{i}{ }^{k} p^{j}\right) \quad \text { and } \quad\left\{p_{i}, x^{j}, x^{4}\right\}_{\lambda}=\lambda^{2} \ell_{s}^{3} R^{4, i j k 4} p_{k}, \\
& \left\{p_{i}, p_{j}, x^{k}\right\}_{\lambda}=-\lambda^{2} \varepsilon_{i j}{ }^{k} x^{4}-\lambda\left(\delta_{j}{ }^{k} x_{i}-\delta_{i}{ }^{k} x_{j}\right) \quad \text { and } \quad\left\{p_{i}, p_{j}, x^{3}\right\}_{\lambda}=\lambda^{3} \varepsilon_{i j k} x^{k}, \\
& \left\{p_{i}, p_{j}, p_{k}\right\}_{\lambda}=0 .
\end{aligned}
$$

These brackets also admit a natural description as an $L_{\infty}$-algebra which is discussed in [93]. Note that the brackets involving solely the momentum coordinates $p_{i}$ correspond to a quaternion subalgebra.

In fact, these brackets originate from the nonassociative alternative algebra of octonions $\mathbb{O}$ which we discussed in Section 1.2 through the redefinition of the imaginary unit octonions given by

$$
\vec{X}=\left(X^{A}\right)=\left(x^{i}, x^{4}, p_{i}\right)=\Lambda\left(e_{A}\right)=\frac{1}{2}\left(\sqrt{\lambda \ell_{s}^{3} R / 3} f_{i}, \sqrt{\lambda^{3} \ell_{s}^{3} R / 3} e_{7},-\lambda e_{i}\right) .
$$

The crucial observation of [60] is that at $\lambda=0$ these brackets reduce to those of the magnetic Poisson brackets for the six-dimensional phase space of closed strings in the $R$-flux background (with the M-theory circle coordinate $x^{4}=1$ becoming a central element of the algebra). Since the contraction parameter $\lambda$ corresponds to the radius of the M-theory circle, this limit is the weak coupling limit $g_{s} \rightarrow 0$ which reduces M-theory to perturbative type IIA string theory in the $R$-flux background.

\subsection{Quantization of the M2-brane phase space}

The quantization of the quasi-Poisson brackets (4.6) of the M2-brane phase space was carried out in [61]. It is based on the notion of a $G_{2}$-structure which stems from the automorphism group of the algebra of octonions $\mathbb{O}$. Given the octonionic structure constants $\eta_{A B C}$ from (1.4), we equip the seven-dimensional real vector space $\mathbb{R}^{7}$ with the cross product

$$
\left(\vec{K} \times{ }_{\eta} \vec{P}\right)_{A}=\eta_{A B C} K_{B} P_{C},
$$

for vectors $\vec{K}=\left(K_{A}\right)$ and $\vec{P}=\left(P_{A}\right)$ in $\mathbb{R}^{7}$. This extends the usual cross product $\times$ on $\mathbb{R}^{3}$ defined analogously in terms of the quaternionic structure constants $\varepsilon_{i j k}$, whose properties can be abstracted into a general set of axioms independent of dimension [45,61]; similarly to the pattern followed by the real normed division algebras, which only exist in dimensions 1,2, 4 and 8, cross products only exist on vector spaces of real dimensions $0,1,3$ and 7 (where in the first two instances the cross product of two vectors is defined to be 0 ). The cross product (4.9) is invariant under the subgroup $G_{2} \subset S O(7)$ of the rotation group of $\mathbb{R}^{7}$. A $G_{2}$-structure on a seven-dimensional real vector space is a cross product that can be brought to the form (4.9) by a suitable change of basis.

The cross product has a natural representation on the octonion algebra $\mathbb{O}$. Given a vector $\vec{K} \in \mathbb{R}^{7}$, define the octonion element

$$
O_{\vec{K}}=K^{A} e_{A}
$$


Then the cross product (4.9) naturally appears as the closure condition of these elements under the octonionic commutator:

$$
O_{\vec{K} \times \eta \vec{P}}=\frac{1}{2}\left[O_{\vec{K}}, O_{\vec{P}}\right] .
$$

Using the alternativity property $(O O) O=O(O O)$ for all $O \in \mathbb{O}$, we can unambiguously define powers of any octonion, and via formal power series expansion an octonionic exponential $\exp (O)$. For the elements (4.10), one may use the unit octonion relations (1.4) to explicitly compute

$$
\exp \left(O_{\vec{K}}\right)=\cos |\vec{K}|+\frac{\sin |\vec{K}|}{|\vec{K}|} O_{\vec{K}}
$$

by a calculation completely analogous to the familiar one in quantum mechanics which uses the quaternion relations of the Pauli spin matrices. From (4.12) one can now compute explicitly the octonionic Baker-Campbell-Hausdorff formula

$$
\exp \left(O_{\vec{K}}\right) \exp \left(O_{\vec{P}}\right)=\exp \left(O_{\overrightarrow{\mathscr{B}}_{\eta}(\vec{K}, \vec{P})}\right)
$$

where the expression for the composition element $\overrightarrow{\mathscr{B}}_{\eta}(\vec{K}, \vec{P})$ is somewhat complicated, see [61] for its explicit form. With it one can construct an octonionic Weyl correspondence $\Delta_{\mathscr{O}}(\vec{X})$ between functions on the seven-dimensional M2-brane phase space and octonions, and by pulling back the octonionic products in the usual way one derives a noncommutative and nonassociative star product of functions $f, g \in C^{\infty}\left(\mathscr{M}^{\prime}\right)$ defined via a twisted Fourier convolution product [61]

$$
\left(f \star_{\lambda} g\right)(\vec{X})=\int_{\tilde{\mathscr{M}}^{\prime}} \int_{\tilde{\mathscr{M}}^{\prime}} \tilde{f}(\vec{K}) \tilde{g}(\vec{P}) \mathrm{e}^{\mathrm{i} \hbar \overrightarrow{\mathscr{B}}_{\eta}(\Lambda \vec{K}, \Lambda \vec{P}) \cdot \Lambda^{-1} \vec{X}} \frac{\mathrm{d} \vec{K}}{(2 \pi)^{7}} \frac{\mathrm{d} \vec{P}}{(2 \pi)^{7}},
$$

where the nondegenerate $7 \times 7$ transformation matrix $\Lambda$ is defined in (4.8). This star product has the non-trivial feature that in the contraction limit it suitably reduces to the star product of the string $R$-flux model [61]:

$$
\lim _{\lambda \rightarrow 0}\left(f \star_{\lambda} g\right)(\vec{X})=\left(f \star_{R} g\right)(X)
$$

after setting the central element $x^{4}=1$.

To understand the spacetime significance of this nonassociative phase space star product, let us introduce the M2-brane triproducts via projection to the four-dimensional configuration space similarly to before:

$$
\left(f_{1} \Delta_{\lambda} f_{2} \Delta_{\lambda} f_{3}\right)(\vec{x})=\left.\left(\left(f_{1} \star_{\lambda} f_{2}\right) \star_{\lambda} f_{3}\right)\left(x^{\mu}, p_{i}\right)\right|_{p=0}
$$

for any three functions $f_{1}, f_{2}, f_{3} \in C^{\infty}\left(M^{\prime}\right)$. Again the explicit form is much more cumbersome and complicated compared to the string theory triproducts, see [61] for the technical details. Defining 3 -brackets via antisymmetrization of these triproducts as in (3.10), for $\lambda=1$ one finds that the 3-brackets of local configuration space coordinates are given by

$$
\left[x^{\mu}, x^{v}, x^{\alpha}\right]_{\Delta_{1}}=\hbar^{2} \ell_{s}^{3} R \varepsilon^{\mu v \alpha \beta} x^{\beta}
$$


which we recognise as a quantization of the defining relations (up to rescaling) of the 3-Lie algebra $A_{4}$ which appears as the nonassociative gauge symmetry underlying the theory of a pair of M2branes in the Bagger-Lambert theory [33]. The classical version of (4.17) defines, after restriction to vectors of unit length $|\vec{x}|=1$, the canonical $S O$ (4)-invariant Nambu-Poisson 3-bracket on the 3sphere $S^{3}$ [11]. Thus the triproducts in this case provide a definition of a quantum 3-sphere, which as discussed in Section 1.6 is an important missing ingredient in the understanding of the dynamics of M5-branes in M-theory. These triproducts are naturally inherited from the nonassociative star product on the seven-dimensional M2-brane phase space in the locally non-geometric background.

\section{M-waves in non-geometric Kaluza-Klein monopole backgrounds}

\subsection{Magnetic monopoles in quantum gravity}

Let us recap where we have gotten to in the present exposition. In Section 2 we considered the magnetic Poisson brackets describing the phase space kinematics of electrically charged particles in magnetic monopole backgrounds. In Section 3 we then applied Born reciprocity to map these brackets onto those describing the phase space of closed strings in $R$-flux backgrounds, and in Section 4 we lifted these brackets to the phase space of M2-branes. In this final section we ask what is the fate of the M2-brane phase space kinematics when we reapply Born reciprocity.

For this, we apply again an order 4 transformation $\left(x^{\mu}, p_{i}\right) \mapsto\left(p_{\mu},-x^{i}\right)$ on $\mathscr{M}^{\prime}$, together with the substitution of $\ell_{s}^{3} R$ with $e \rho$, to the brackets (4.6) to get

$$
\begin{aligned}
& \left\{x^{i}, x^{j}\right\}_{\rho}=\lambda \varepsilon^{i j k} x^{k}, \\
& \left\{p_{i}, p_{j}\right\}_{\rho}=-e \rho \varepsilon_{i j k} x^{k} \quad \text { and } \quad\left\{p_{4}, p_{i}\right\}_{\rho}=-\lambda e \rho x_{i}, \\
& \left\{x_{i}, p_{j}\right\}_{\rho}=-\delta_{i j} p_{4}-\lambda \varepsilon_{i j k} p_{k} \quad \text { and } \quad\left\{x^{i}, p_{4}\right\}_{\rho}=\lambda^{2} x^{i} .
\end{aligned}
$$

When $\lambda=0$, these reduce to the magnetic Poisson brackets (2.4) for an electric charge $e$ in a uniform distribution $\rho$ of magnetic charge on $\mathbb{R}^{3}$ with the rotationally symmetric magnetic field (2.16). For generic values of $\lambda$, the brackets (5.1) describe the kinematics of a seven-dimensional phase space with an "extra" momentum mode $p_{4}$. We would now like to understand what physics this system represents.

Setting $\rho=0$ in (5.1) reveals that the brackets describe a noncommutative but associative deformation of spacetime whose coordinates $x^{i}$ generate a quaternion subalgebra with commuting momenta

$$
\left\{p_{i}, p_{j}\right\}_{0}=\left\{p_{i}, p_{4}\right\}_{0}=0 .
$$

The quantity $\lambda^{2} \vec{p}^{2}+p_{4}^{2}$ is easily computed to be a central element of the algebra (5.1) for $\rho=0$, so without loss of generality we may restrict the four-dimensional momentum space to the surface of the ellipsoid

$$
\lambda^{2} \vec{p}^{2}+p_{4}^{2}=1 .
$$

Since the momentum $p_{4}$ commutes with the other momenta, we can solve this equation for $p_{4}$ on the upper hemisphere of the ellipsoid and reduce the remaining brackets of (5.1) to

$$
\left\{x^{i}, x^{j}\right\}_{0}=\lambda \varepsilon^{i j k} x^{k} \quad \text { and } \quad\left\{x_{i}, p_{j}\right\}_{0}=-\sqrt{1-\lambda^{2} \vec{p}^{2}} \delta_{i j}-\lambda \varepsilon_{i j k} p_{k} .
$$


For $\lambda=0$ these are just the canonical phase space Poisson brackets of the configuration space $\mathbb{R}^{3}$. For $\lambda \neq 0$, they are the natural invariant Poisson brackets on the six-dimensional phase space whose momentum coordinates live on the ellipsoid (5.3), with the conjugate coordinates $x^{i}$ acting as covariant derivatives. The quantization of these brackets has appeared before in the context of a Ponzano-Regge spin foam model of three-dimensional quantum gravity [108], provided the parameter $\lambda$ is identified with the Planck length $\ell_{\mathrm{P}}$ of the three-dimensional spacetime through

$$
\lambda=\frac{\ell_{\mathrm{P}}}{\hbar} \text {. }
$$

Combining these observations together, we may infer that the uncontracted octonion algebra, after Born reciprocity, is in some sense related to the dynamics of magnetic monopoles in the spacetime of three-dimensional quantum gravity with the identification (5.5). The precise gravitational system which realises this situation was recognised by [63] in the setting of locally non-geometric M-theory, and we shall now turn to some of the details.

\subsection{The M-wave phase space}

A magnetic monopole can be embedded into type IIA string theory as a D6-brane, with the electric probes provided by D0-branes inside the D6-brane. The D6-brane lifts to M-theory as a Kaluza-Klein monopole, which is a background of 11-dimensional supergravity given by the metric

$$
\mathrm{d} s_{11}^{2}=\mathrm{d} s_{7}^{2}+U \mathrm{~d} \vec{x} \cdot \mathrm{d} \vec{x}+U^{-1}\left(\mathrm{~d} x^{4}+\vec{A} \cdot \mathrm{d} \vec{x}\right)^{2}
$$

where $\mathrm{d} s_{7}^{2}$ is the metric of the transverse space to the M-theory compactification to four dimensions. The three-dimensional vector potential $\vec{A}(\vec{x})$ and the harmonic function $U(\vec{x})$ are related through

$$
\vec{\nabla} \times \vec{A}=\vec{\nabla} U \quad \text { and } \quad \vec{\nabla}^{2} U=\rho,
$$

for a given distribution of magnetic charge $\rho$. The electric probes of this background are M-waves along the M-theory circle $x^{4} \in S^{1}$, which lift the D0-branes.

The standard Kaluza-Klein monopole solution corresponds to the embedding of a single Dirac monopole, with singular distribution $\rho(\vec{x})=4 \pi g \delta^{(3)}(\vec{x})$, which lifts a single D6-brane. In that case the vector potential $\vec{A}=\vec{A}_{g, \vec{n}}$ is given locally by (2.10) and the harmonic function $U(\vec{x})$ is given by the Green's function of the three-dimensional Laplacian. Then the four-dimensional part of the metric (5.6) is just the standard Taub-NUT metric.

To understand how the purported locally non-geometric background fits into this solution, we first observe that the parameters of type IIA string theory, the string coupling $g_{s}$ and the string length $\ell_{s}$, are related to the parameters of M-theory, the 11-dimensional Planck length $\ell_{\mathrm{P}}$ and the radius of the M-theory circle $R_{11}$, through the identifications [109]

$$
\ell_{s}^{2}=\frac{\ell_{\mathrm{P}}^{3}}{R_{11}} \quad \text { and } \quad g_{s}=\left(\frac{R_{11}}{\ell_{\mathrm{P}}}\right)^{3 / 2} .
$$

The proper reduction which takes M-theory to type IIA string theory is the limit where $g_{s} \rightarrow$ 0 and $R_{11} \rightarrow 0$ with $\ell_{s}$ finite. This is compatible with the identification (5.5) of the algebraic contraction parameter $\lambda$ as the Planck length, since then the limit corresponds to $\lambda \sim \ell_{\mathrm{P}} \sim R_{11}^{1 / 3} \rightarrow 0$, as required. 
In the present case we are interested in a uniform distribution of magnetic charge $\rho$, which can be understood as a smearing of Dirac monopoles throughout three-dimensional space. In this instance there is no local expression for the vector potential $\vec{A}$ or the metric (5.6), see [63] for a formal non-local expression. This smeared solution is called a non-geometric Kaluza-Klein monopole, which lifts a uniform distribution of D6-branes in type IIA string theory. Geometrically, it corresponds to the passage from the four-dimensional Taub-NUT space, realised as the total space of an $S^{1}$-bundle over $\mathbb{R}^{3}$, to the total space of an $S^{1}$-gerbe over $\mathbb{R}^{3}$ which realizes the constant magnetic charge [63], similarly to our discusson of geometric quantization in Section 2.5. This corroborates the interpretation of the brackets (5.1) as governing the phase space dynamics of the M-waves; that the phase space is now lacking a position coordinate stems from the fact that the wave has no well-defined localised position $x^{4} \in S^{1}$.

\subsection{The covariant M-theory phase space 3-algebra}

In [61] it was proposed to view the full eight-dimensional phase space $\hat{\mathscr{M}}^{\prime}$ of the M-theory compactification to four dimensions, with or without $R$-flux or Kaluza-Klein monopole charge, in terms of a natural 3-algebra structure, akin to those expected to arise in M-theory that we discussed in Section 1.6. In [63] it was then subsequently shown how this 3-algebra provides a unified description of the M2-brane and M-wave phase spaces. The starting point is the notion of a $\operatorname{Spin}(7)$ structure which is defined by collecting all eight generators of the octonion algebra $\mathbb{O}$ into $\xi_{\hat{A}}=$ $\left(\xi_{0}, \vec{\xi}\right)=\left(1, e_{A}\right)$ and defining the 3 -brackets

$$
\left\{\xi_{\hat{A}}, \xi_{\hat{B}}, \xi_{\hat{C}}\right\}_{\phi}=\phi_{\hat{A} \hat{B} \hat{C} \hat{D}} \xi_{\hat{D}}
$$

where the self-dual 4-form $\phi$ has components

$$
\phi_{0 A B C}=\eta_{A B C} \quad \text { and } \quad \phi_{A B C D}=\eta_{A B C D}
$$

given by the structure constants $\eta_{A B C}$ of the octonionic commutators (1.4) and the structure constants $\eta_{A B C D}$ of the octonionic Jacobiators (1.6). The 3-brackets (5.9) are symmetric under the subgroup $\operatorname{Spin}(7) \subset S O(8)$ of the eight-dimensional rotation group and define a ternary operation called a triple cross product on the real vector space $\mathbb{R}^{8}$ [45,61]. A $\operatorname{Spin}(7)$-structure on an eightdimensional real vector space is a triple cross product that can be brought to the form (5.9) by a suitable change of basis.

Let us now map the 3-brackets (5.9) onto 3-brackets for the eight-dimensional phase space coordinates through the redefinition of the generators of $\mathbb{O}$ given by

$$
X^{\hat{A}}=\left(x^{\mu}, p_{\mu}\right)=\left(\Lambda \vec{\xi},-\frac{\lambda}{2} \xi_{0}\right)
$$

where the invertible $7 \times 7$ transformation matrix $\Lambda$ is defined in (4.8). This splitting breaks the 
$\operatorname{Spin}(7)$-symmetry to $S O(4) \times S O(4)$ and results in the 3-brackets

$$
\begin{aligned}
& \left\{x^{i}, x^{j}, x^{k}\right\}_{\phi}=-\frac{\ell_{s}^{3}}{2} R^{4, i j k 4} x^{4} \quad \text { and } \quad\left\{x^{i}, x^{j}, x^{4}\right\}_{\phi}=\frac{\lambda^{2} \ell_{s}^{3}}{2} R^{4, i j k 4} x_{k}, \\
& \left\{p^{i}, x^{j}, x^{k}\right\}_{\phi}=-\frac{\lambda^{2} \ell_{s}^{3}}{2} R^{4, i j k 4} p_{4}-\frac{\lambda \ell_{s}^{3}}{2} R^{4, i j k 4} p_{k}, \\
& \left\{p_{i}, x^{j}, x^{4}\right\}_{\phi}=-\frac{\lambda^{2} \ell_{s}^{3}}{2} R^{4,1234} \delta_{i}^{j} p_{4}-\frac{\lambda^{2} \ell_{s}^{3}}{2} R^{4, i j k 4} p_{k}, \\
& \left\{p_{i}, p_{j}, x^{k}\right\}_{\phi}=\frac{\lambda^{2}}{2} \varepsilon_{i j}^{k} x^{4}-\frac{\lambda}{2}\left(\delta_{j}^{k} x_{i}-\delta_{i}^{k} x_{j}\right) \\
& \left\{p_{i}, p_{j}, x^{4}\right\}_{\phi}=\frac{\lambda^{3}}{2} \varepsilon_{i j k} x^{k} \quad \text { and } \quad\left\{p_{i}, p_{j}, p_{k}\right\}_{\phi}=2 \lambda \varepsilon_{i j k} p_{4} \text {, } \\
& \left\{p_{4}, x^{i}, x^{j}\right\}_{\phi}=\frac{\lambda \ell_{s}^{3}}{2} R^{4, i j k 4} p_{k} \quad \text { and } \quad\left\{p_{4}, x^{i}, x^{4}\right\}_{\phi}=-\frac{\lambda^{2} \ell_{s}^{3}}{2} R^{4,1234} p^{i} \\
& \left\{p_{4}, p_{i}, x^{j}\right\}_{\phi}=\frac{\lambda}{2} \delta_{i}^{j} x^{4}+\frac{\lambda^{2}}{2} \varepsilon_{i}^{j k} x_{k} \\
& \left\{p_{4}, p_{i}, x^{4}\right\}_{\phi}=\frac{\lambda^{3}}{2} x_{i} \quad \text { and } \quad\left\{p_{4}, p_{i}, p_{j}\right\}_{\phi}=\frac{\lambda^{2}}{2} \varepsilon_{i j k} p^{k}
\end{aligned}
$$

The first set of 3-brackets in (5.12) for $\lambda=1$ are just the brackets of the 3-Lie algebra $A_{4}$ (up to rescaling) that we encountered in (4.17), which reduce to the Jacobiators (3.2) of the string $R$-flux model when $x^{4}=1$ is central. These 3-brackets even describe the free eight-dimensional M-theory phase space in the absence of $R$-flux or Kaluza-Klein monopole charge, in which case only the 3-brackets (5.15), (5.16), (5.18) and (5.19) are non-zero. These 3-brackets do not define a NambuPoisson bracket, because the fundamental identity (1.14) does not hold and its failure is controlled by a higher 5 -bracket $[61,63]$. They all vanish in the limit $\lambda \sim \ell_{\mathrm{P}} \rightarrow 0$ where quantum gravitational effects are turned off.

Consider now any constraint

$$
G\left(X^{\hat{1}}, \ldots, X^{\hat{8}}\right)=0
$$

on the eight-dimensional phase space $\hat{\mathscr{M}}^{\prime}$. It naturally defines a 2-bracket

$$
\{f, g\}_{G}:=\{f, g, G\}_{\phi}
$$

on functions $f, g \in C^{\infty}\left(\hat{\mathscr{M}}^{\prime}\right)$, which describes the reduction of $\hat{\mathscr{M}}^{\prime}$ with its 3 -algebra to a sevendimensional submanifold with quasi-Poisson brackets (5.21). This breaks the $\operatorname{Spin}(7)$-symmetry to its $G_{2}$ subgroup, under which the spinor representation of $\operatorname{Spin}(7)$ decomposes according to the branching rule

$$
\left.8\right|_{G_{2}}=7 \oplus 1
$$

appropriate to the reduction to a seven-dimensional subspace.

For example, the constraint function $G\left(x^{\mu}, p_{\mu}\right)=p_{4}$ gives the M2-brane phase space of Section 4 with the quasi-Poisson brackets (4.6). On the other hand, the choice $G\left(x^{\mu}, p_{\mu}\right)=x^{4}$ gives the 
M-wave phase space of this section with the quasi-Poisson brackets (5.1) (under the identification $\left.\ell_{s}^{3} R=e \rho\right)$. The two phase spaces are just different seven-dimensional slices of the same eightdimensional phase space $\hat{\mathscr{M}}^{\prime}$ and are related by Born reciprocity $\left(x_{\mu}, p^{\mu}\right) \mapsto\left(p_{\mu},-x^{\mu}\right)$, which is realised here as a $\operatorname{Spin}(7)$-transformation. Other linear seven-dimensional slices of the eightdimensional M-theory phase space $\hat{\mathscr{M}}^{\prime}$ are likewise related by rotations valued in $\operatorname{Spin}(7) \subset S O(8)$.

\section{Acknowledgments}

We would like to thank the organisors of the Workshop on "Dualities and Generalized Geometries" for the invitation to deliver these lectures, and all the participants for their questions and comments which have helped shape the structure and content of this article. We are particularly grateful to David Berman, Bernd Henschenmacher, Jeffrey Heninger, Chris Hull, Vladislav Kupriyanov, Dieter Lüst, Nick Mavromatos and Philip Morrison for discussions and correspondence on the contents of this paper. This work was supported in part by the Action MP1405 QSPACE from the European Cooperation in Science and Technology (COST) and by the Consolidated Grant ST/P000363/1 from the UK Science and Technology Facilities Council (STFC).

\section{References}

[1] R.D. Schafer, An Introduction to Nonassociative Algebras (Dover Publications, New York, 1995).

[2] J.C. Baez, “The octonions,” Bull. Amer. Math. Soc. 39, 145-205 (2002) [Erratum: Bull. Am. Math. Soc. 42, 213 (2005)] [arXiv:math-ra/0105155].

[3] P. Jordan, “Über Eine Klasse Nichtassociativer Hyperkomplexer Algebren,” Nachr. Ges. Wiss. Göttingen, 569-575 (1932).

[4] A.A. Albert, “On Jordan algebras of linear transformations," Trans. Amer. Math. Soc. 59, 524-555 (1946).

[5] R.D. Schafer, "Structure and representation of nonassociative algebras," Bull. Amer. Math. Soc. 61, 469-484 (1955).

[6] P. Jordan, J. von Neumann and E. Wigner, "On an algebraic generalization of the quantum mechanical formalism,” Ann. Math. 35, 29-64 (1934).

[7] M. Günaydin, C. Piron and H. Ruegg, "Moufang plane and octonionic quantum mechanics," Commun. Math. Phys. 61, 69-85 (1978).

[8] E.I. Zelmanov, “On prime Jordan algebras II,” Sibirsk Mat. J. 24, 89-104 (1983).

[9] Y. Nambu, “Generalized Hamiltonian dynamics,” Phys. Rev. D 7, 2405-2412 (1973).

[10] L. Takhtajan, "On foundation of the generalized Nambu mechanics," Commun. Math. Phys. 160, 295-316 (1994) [arXiv:hep-th/9301111].

[11] J. DeBellis, C. Saemann and R.J. Szabo, "Quantized Nambu-Poisson manifolds and n-Lie algebras," J. Math. Phys. 51, 122303 (2010) [arXiv:1001.3275 [hep-th]].

[12] E. Witten, “Noncommutative geometry and string field theory," Nucl. Phys. B 268, 253-294 (1986).

[13] A. Strominger, “Closed string field theory,” Nucl. Phys. B 294, 93-112 (1987). 
[14] G.T. Horowitz and A. Strominger, "Translations as inner derivations and associativity anomalies in open string field theory," Phys. Lett. B 185, 45-51 (1987).

[15] B. Zwiebach, "Closed string field theory: Quantum action and the BV master equation," Nucl. Phys. B 390, 33-152 (1993) [arXiv:hep-th/9206084].

[16] T. Lada and J. Stasheff, “Introduction to sh Lie algebras for physicists,” Int. J. Theor. Phys. 32, 1087-1104 (1993) [arXiv:hep-th/9209099].

[17] J. Stasheff, "Closed string field theory, strong homotopy Lie algebras and the operad actions of moduli space," in: Perspectives in Mathematical Physics, eds. R. Penner and S.-T. Yau (International Press, Boston), 265-288 (1994) [arXiv:hep-th/9304061].

[18] J. Stasheff, “ $L_{\infty}$ and $A_{\infty}$ structures: Then and now,” arXiv:1809.02526 [math.QA].

[19] N. Seiberg and E. Witten, "String theory and noncommutative geometry," JHEP 9909, 032 (1999) [arXiv:hep-th/9908142].

[20] M.R. Douglas and N.A. Nekrasov, "Noncommutative field theory," Rev. Mod. Phys. 73, 977-1029 (2001) [arXiv:hep-th/0106048].

[21] R.J. Szabo, “Quantum field theory on noncommutative spaces,” Phys. Rept. 378, 207-299 (2003) [arXiv:hep-th/0109162].

[22] L. Cornalba and R. Schiappa, "Nonassociative star product deformations for D-brane worldvolumes in curved backgrounds," Commun. Math. Phys. 225, 33-66 (2002) [arXiv:hep-th/0101219].

[23] M. Herbst, A. Kling and M. Kreuzer, "Star products from open strings in curved backgrounds," JHEP 0109, 014 (2001) [arXiv:hep-th/0106159].

[24] M. Herbst, A. Kling and M. Kreuzer, “Cyclicity of nonassociative products on D-branes,” JHEP 0403, 003 (2004) [arXiv:hep-th/0312043].

[25] R.J. Szabo, "Symmetry, gravity and noncommutativity," Class. Quant. Grav. 23, R199-R242 (2006) [arXiv:hep-th/0606233].

[26] R. Blumenhagen, I. Brunner, V.G. Kupriyanov and D. Lüst, "Bootstrapping noncommutative gauge theories from $L_{\infty}$-algebras,” JHEP 1805, 097 (2018) [arXiv:1803.00732 [hep-th]].

[27] V. Mathai and J.M. Rosenberg, "T-duality for torus bundles with $H$-fluxes via noncommutative topology," Commun. Math. Phys. 253, 705-721 (2004) [arXiv:hep-th/0401168].

[28] P. Bouwknegt, K. Hannabuss and V. Mathai, "Nonassociative tori and applications to T-duality," Commun. Math. Phys. 264, 41-69 (2006) [arXiv:hep-th/0412092].

[29] J. Brodzki, V. Mathai, J.M. Rosenberg and R.J. Szabo, "D-branes, RR-fields and duality on noncommutative manifolds,” Commun. Math. Phys. 277, 643-706 (2008) [arXiv:hep-th/0607020].

[30] A. Basu and J.A. Harvey, “The M2-M5-brane system and a generalized Nahm's equation," Nucl. Phys. B 713, 136-150 (2005) [arXiv:hep-th/0412310].

[31] J. Bagger and N. Lambert, “Modeling multiple M2's,” Phys. Rev. D 75, 045020 (2007) [arXiv:hep-th/0611108].

[32] J. Bagger and N. Lambert, "Gauge symmetry and supersymmetry of multiple M2-branes," Phys. Rev. D 77, 065008 (2008) [arXiv:0711.0955 [hep-th]].

[33] J. Bagger, N. Lambert, S. Mukhi and C. Papageorgakis, "Multiple membranes in M-theory,” Phys. Rept. 527, 1-100 (2013) [arXiv:1203.3546 [hep-th]]. 
[34] J. DeBellis, C. Saemann and R.J. Szabo, "Quantized Nambu-Poisson manifolds in a 3-Lie algebra reduced model,” JHEP 1104, 075 (2011) [arXiv:1012.2236 [hep-th]].

[35] J. DeBellis and R.J. Szabo, "Reduced Chern-Simons quiver theories and cohomological 3-algebra models," PTEP 2013, 113B01 (2013) [arXiv:1307.1663 [hep-th]].

[36] C. Saemann, "Lectures on higher structures in M-theory," in: Noncommutative Geometry and Physics 4, eds. Y. Maeda, H. Moriyoshi, M. Kotani and S. Watamura (World Scientific Publishing), 171-210 (2017) [arXiv:1609.09815 [hep-th]].

[37] E. Bergshoeff, D.S. Berman, J.P. van der Schaar and P. Sundell, “A noncommutative M-theory 5-brane,” Nucl. Phys. B 590, 173-197 (2000) [arXiv:hep-th/0005026].

[38] S. Kawamoto and N. Sasakura, "Open membranes in a constant $C$-field background and noncommutative boundary strings,” JHEP 0007, 014 (2000) [arXiv:hep-th/0005123].

[39] C.-S. Chu and D.J. Smith, "Towards the quantum geometry of the M5-brane in a constant $C$-field from multiple membranes," JHEP 0904, 097 (2009) [arXiv:0901.1847 [hep-th]].

[40] C. Saemann and R.J. Szabo, “Groupoid quantization of loop spaces,” PoS CORFU 2011, 046 (2011) [arXiv:1203.5921 [hep-th]].

[41] C. Saemann and R.J. Szabo, "Groupoids, loop spaces and quantization of 2-plectic manifolds," Rev. Math. Phys. 25, 1330005 (2013) [arXiv:1211.0395 [hep-th]].

[42] P.-M. Ho and Y. Matsuo, “The Nambu bracket and M-theory,” PTEP 2016, 06A104 (2016) [arXiv:1603.09534 [hep-th]].

[43] B.S. Acharya, "M-theory, $G_{2}$-manifolds and four-dimensional physics," Class. Quant. Grav. 19, 5619-5653 (2002).

[44] S. Gukov and J. Sparks, “M-theory on Spin(7)-manifolds 1,” Nucl. Phys. B 625, 3-69 (2002) [arXiv:hep-th/0109025].

[45] D.A. Salamon and T. Walpuski, "Notes on the octonions," Proc. 23rd Gokova Geom.-Topol. Conf. (2017), 1-85 [arXiv:1005.2820 [math.RA]].

[46] R. Jackiw, “3-cocycle in mathematics and physics,” Phys. Rev. Lett. 54, 159-162 (1985).

[47] M. Günaydin and B. Zumino, "Magnetic charge and nonassociative algebras," in: Old and New Problems in Fundamental Physics: Meeting in Honour of G.C. Wick (Quaderni, Pisa: Scuola Normale Superiore), 43-53 (1986).

[48] L.D. Faddeev and S.L. Shatashvili, "Algebraic and Hamiltonian methods in the theory of nonabelian anomalies," Theor. Math. Phys. 60, 770-778 (1985).

[49] L.D. Faddeev and S.L. Shatashvili, "Realization of the Schwinger term in the Gauss law and the possibility of correct quantization of a theory with anomalies," Phys. Lett. B 167, 225-228 (1986).

[50] G.W. Semenoff, "Nonassociative electric fields in chiral gauge theory: An explicit construction," Phys. Rev. Lett. 60, 680-683 (1988) [Erratum: Phys. Rev. Lett. 60, 1590 (1988)].

[51] J. Brodzki, V. Mathai, J.M. Rosenberg and R.J. Szabo, "Noncommutative correspondences, duality and D-branes in bivariant K-theory,” Adv. Theor. Math. Phys. 13, 497-552 (2009) [arXiv:0708.2648 [hep-th]].

[52] R. Blumenhagen and E. Plauschinn, "Nonassociative gravity in string theory?," J. Phys. A 44, 015401 (2011) [arXiv:1010.1263 [hep-th]]. 
[53] D. Lüst, "T-duality and closed string noncommutative (doubled) geometry," JHEP 1012, 084 (2010) [arXiv:1010.1361 [hep-th]].

[54] R. Blumenhagen, A. Deser, D. Lüst, E. Plauschinn and F. Rennecke, "Non-geometric fluxes, asymmetric strings and nonassociative geometry," J. Phys. A 44, 385401 (2011) [arXiv:1106.0316 [hep-th]].

[55] D. Mylonas, P. Schupp and R.J. Szabo, "Membrane sigma-models and quantization of non-geometric flux backgrounds," JHEP 1209, 012 (2012) [arXiv:1207.0926 [hep-th]].

[56] A. Chatzistavrakidis and L. Jonke, "Matrix theory origins of non-geometric fluxes," JHEP 1302, 040 (2013) [arXiv:1207.6412 [hep-th]].

[57] R. Blumenhagen, M. Fuchs, F. Haßler, D. Lüst and R. Sun, "Nonassociative deformations of geometry in double field theory," JHEP 1404, 141 (2014) [arXiv:1312.0719 [hep-th]].

[58] C.D.A. Blair, "Noncommutativity and nonassociativity of the doubled string in non-geometric backgrounds," JHEP 1506, 091 (2015) [arXiv:1405.2283 [hep-th]].

[59] L. Freidel, R.G. Leigh and D. Minic, "Noncommutativity of closed string zero modes," Phys. Rev. D 96, 066003 (2017) [arXiv:1707.00312 [hep-th]].

[60] M. Günaydin, D. Lüst and E. Malek, "Nonassociativity in non-geometric string and M-theory backgrounds, the algebra of octonions, and missing momentum modes," JHEP 1611, 027 (2016) [arXiv:1607.06474 [hep-th]].

[61] V.G. Kupriyanov and R.J. Szabo, " $G_{2}$-structures and quantization of non-geometric M-theory backgrounds," JHEP 1702, 099 (2017) [arXiv:1701.02574 [hep-th]].

[62] R.J. Szabo, "Magnetic backgrounds and noncommutative field theory," Int. J. Mod. Phys. A 19, 1837-1862 (2004) [arXiv:physics/0401142].

[63] D. Lüst, E. Malek and R.J. Szabo, "Non-geometric Kaluza-Klein monopoles and magnetic duals of M-theory $R$-flux backgrounds," JHEP 1710, 144 (2017) [arXiv:1705.09639 [hep-th]].

[64] E. Plauschinn, "Non-geometric backgrounds in string theory," arXiv:1811.11203 [hep-th].

[65] R.J. Szabo, "Higher quantum geometry and non-geometric string theory," PoS CORFU 2017, 151 (2018) [arXiv:1803.08861 [hep-th]].

[66] R.J. Szabo, "Quantization of magnetic Poisson structures," arXiv:1903.02845 [hep-th].

[67] C. Klimčík and T. Strobl, "WZW-Poisson manifolds," J. Geom. Phys. 43, 341-344 (2002) [arXiv:math-sg/0104189].

[68] P. Ševera and A. Weinstein, "Poisson geometry with a 3-form background," Prog. Theor. Phys. Suppl. 144, 145-154 (2001) [arXiv:math-sg/0107133].

[69] C. Castelnovo, R. Moessner and S.L. Sondhi, "Magnetic monopoles in spin ice," Nature 451N7174, 42-45 (2008) [arXiv:0710.5515 [cond-mat.str-el]].

[70] D.J.P. Morris et al., "Dirac strings and magnetic monopoles in spin ice Dy2Ti2O7," Science 326, 411-414 (2009) [arXiv:1011.1174 [cond-mat.mtrl-sci]].

[71] R.J. Szabo, "Magnetic monopoles and nonassociative deformations of quantum theory," J. Phys. Conf. Ser. 965, 012041 (2018) [arXiv:1709.10080 [hep-th]].

[72] I. Bakas and D. Lüst, "3-cocycles, nonassociative star products and the magnetic paradigm of $R$-flux string vacua," JHEP 1401, 171 (2014) [arXiv:1309.3172 [hep-th]]. 
[73] J.M. Heninger and P.J. Morrison, "Hamiltonian nature of monopole dynamics," arXiv:1808.08689 [math-ph].

[74] V.G. Kupriyanov and R.J. Szabo, "Symplectic realization of electric charge in fields of monopole distributions,” Phys. Rev. D 98, 045005 (2018) [arXiv:1803.00405 [hep-th]].

[75] S. Bunk, L. Müller and R.J. Szabo, "Geometry and 2-Hilbert space for nonassociative magnetic translations," arXiv:1804.08953 [hep-th].

[76] T.L. Curtright, D.B. Fairlie and C.K. Zachos, A Concise Treatise on Quantum Mechanics in Phase Space (World Scientific Publishing, 2014).

[77] T.T. Wu and C.N. Yang, "Dirac monopole without strings: Monopole harmonics," Nucl. Phys. B 107, 365-380 (1976).

[78] R. Jackiw, “Dirac's magnetic monopoles (again),” Int. J. Mod. Phys. A 19S1, 137-144 (2004) [arXiv:hep-th/0212058].

[79] J.F. Cariñena, J.M. Gracia-Bondía, F. Lizzi, G. Marmo and P. Vitale, "Star product in the presence of a monopole,” Phys. Lett. A 374, 3614-3618 (2010) [arXiv:0912.2197 [math-ph]].

[80] M.A. Soloviev, “Dirac's magnetic monopole and the Kontsevich star product,” J. Phys. A 51, 095205 (2018) [arXiv:1708.05030 [math-ph]].

[81] D. Mylonas, P. Schupp and R.J. Szabo, "Non-geometric fluxes, quasi-Hopf twist deformations and nonassociative quantum mechanics,” J. Math. Phys. 55, 122301 (2014) [arXiv:1312.1621 [hep-th]].

[82] V.G. Kupriyanov and D.V. Vassilevich, "Nonassociative Weyl star products,” JHEP 1509, 103 (2015) [arXiv:1506.02329 [hep-th]].

[83] M. Bojowald, S. Brahma, U. Büyükçam and T. Strobl, "Monopole star products are non-alternative," JHEP 1704, 028 (2017) [arXiv:1610.08359 [math-ph]].

[84] D.V. Vassilevich and F.M.C. Oliveira, "Nearly associative deformation quantization," Lett. Math. Phys. 108, 2293-2301 (2018) [arXiv:1802.05808 [math-ph]].

[85] M. Bojowald, S. Brahma, U. Büyükçam and T. Strobl, "States in nonassociative quantum mechanics: Uncertainty relations and semiclassical evolution,” JHEP 1503, 093 (2015) [arXiv:1411.3710 [hep-th]].

[86] M. Bojowald, S. Brahma and U. Büyükçam, “Testing nonassociative quantum mechanics,” Phys. Rev. Lett. 115, 220402 (2015) [Erratum: Phys. Rev. Lett. 117, 099901 (2016)] [arXiv:1510.07559 [quant-ph]].

[87] M. Bojowald, S. Brahma, U. Büyükçam, J. Guglielmon and M. van Kuppeveld, "Small magnetic charges and monopoles in nonassociative quantum mechanics," Phys. Rev. Lett. 121, 201602 (2018) [arXiv:1810.06540 [hep-th]].

[88] O. Gould, D.L.-J. Ho and A. Rajantie, "Towards Schwinger production of magnetic monopoles in heavy-ion collisions," arXiv:1902.04388 [hep-th].

[89] B.S. Acharya et al. [MoEDAL Collaboration], "Search for magnetic monopoles with the MoEDAL forward trapping detector in $2.11 \mathrm{fb}^{-1}$ of $13 \mathrm{TeV}$ proton-proton collisions at the LHC," Phys. Lett. B 782, 510-516 (2018) [arXiv:1712.09849 [hep-ex]].

[90] S. Baines, N.E. Mavromatos, V.A. Mitsou, J.L. Pinfold and A. Santra, "Monopole production via photon fusion and Drell-Yan processes: MADGRAPH implementation and perturbativity via velocity-dependent coupling and magnetic moment as novel features,” Eur. Phys. J. C 78, 966 (2018) [arXiv:1808.08942 [hep-ph]]. 
[91] C.M. Hull and B. Zwiebach, "Double field theory," JHEP 0909, 099 (2009) [arXiv:0904.4664 [hep-th]].

[92] V.E. Marotta and R.J. Szabo, "Para-Hermitian geometry, dualities and generalized flux backgrounds," Fortschr. Phys. 67, 1800093 (2019) [arXiv:1810.03953 [hep-th]].

[93] O. Hohm, V.G. Kupriyanov, D. Lüst and M. Traube, "Constructions of $L_{\infty}$-algebras and their field theory realizations," Adv. Math. Phys. 2018, 9282905 (2018) [arXiv:1709.10004 [math-ph]].

[94] J. Shelton, W. Taylor and B. Wecht, "Non-geometric flux compactifications," JHEP 0510, 085 (2005) [arXiv:hep-th/0508133].

[95] C.M. Hull, “A geometry for non-geometric string backgrounds," JHEP 0510, 065 (2005) [arXiv:hep-th/0406102].

[96] D. Andriot, O. Hohm, M. Larfors, D. Lüst and P. Patalong, "Non-geometric fluxes in supergravity and double field theory," Fortsch. Phys. 60, 1150-1186 (2012) [arXiv:1204.1979 [hep-th]].

[97] B. Wecht, "Lectures on non-geometric flux compactifications," Class. Quant. Grav. 24, S773-S794 (2007) [arXiv:0708.3984 [hep-th]].

[98] P. Aschieri and R.J. Szabo, "Triproducts, nonassociative star products and geometry of $R$-flux string compactifications," J. Phys. Conf. Ser. 634, 012004 (2015) [arXiv:1504.03915 [hep-th]].

[99] A. Chatzistavrakidis, L. Jonke, F.S. Khoo and R.J. Szabo, "Double field theory and membrane sigma-models," JHEP 1807, 015 (2018) [arXiv:1802.07003 [hep-th]].

[100] G.E. Barnes, A. Schenkel and R.J. Szabo, "Nonassociative geometry in quasi-Hopf representation categories I: Bimodules and their internal homomorphisms," J. Geom. Phys. 89, 111-152 (2015) [arXiv:1409.6331 [math.QA]].

[101] G.E. Barnes, A. Schenkel and R.J. Szabo, "Nonassociative geometry in quasi-Hopf representation categories II: Connections and curvature," J. Geom. Phys. 106, 234-255 (2016) [arXiv:1507.02792 [math.QA]].

[102] G.E. Barnes, A. Schenkel and R.J. Szabo, "Working with nonassociative geometry and field theory," PoS CORFU 2015, 081 (2016) [arXiv:1601.07353 [hep-th]].

[103] R. Blumenhagen and M. Fuchs, "Towards a theory of nonassociative gravity," JHEP 1607, 019 (2016) [arXiv:1604.03253 [hep-th]].

[104] P. Aschieri, M. Dimitrijević Ćirić and R.J. Szabo, "Nonassociative differential geometry and gravity with non-geometric fluxes," JHEP 1802, 036 (2018) [arXiv:1710.11467 [hep-th]].

[105] D. Lüst, E. Malek and M. Syväri, "Locally non-geometric fluxes and missing momenta in M-theory,” JHEP 1801, 050 (2018) [arXiv:1710.05919 [hep-th]].

[106] C.D.A. Blair and E. Malek, "Geometry and fluxes of $S L(5)$ exceptional field theory," JHEP 1503, 144 (2015) [arXiv:1412.0635 [hep-th]].

[107] A. Chatzistavrakidis, L. Jonke, D. Lüst and R.J. Szabo, "Fluxes in exceptional field theory and threebrane sigma-models," arXiv:1901.07775 [hep-th].

[108] L. Freidel and E.R. Livine, " $3 D$ quantum gravity and effective noncommutative quantum field theory,” Phys. Rev. Lett. 96, 221301 (2006) [arXiv:hep-th/0512113].

[109] E. Witten, "String theory dynamics in various dimensions," Nucl. Phys. B 443, 85-126 (1995) [arXiv:hep-th/9503124]. 